\title{
SOFIA/FORCAST Galactic Center Legacy Survey: Overview
}

\author{
Matthew J. Hankins ${ }^{1}$ (D), Ryan M. Lau ${ }^{2}$, James T. Radomski ${ }^{3}$, Angela S. Cotera ${ }^{4}$, Mark R. Morris ${ }^{5}$ (D), Elisabeth A. C. Mills ${ }^{6}$ (D), \\ Daniel L. Walker ${ }^{7,8}$, Ashley T. Barnes ${ }^{9}$, Janet P. Simpson ${ }^{4}$ (D), Terry L. Herter ${ }^{10}$ (D), Steven N. Longmore ${ }^{11}$ (D), John Bally ${ }^{12}$ (D), \\ Mansi M. Kasliwal ${ }^{1}$ (D), Nadeen B. Sabha ${ }^{13}$, and Macarena García-Marin ${ }^{14}$ \\ ${ }^{1}$ Division of Physics, Mathematics, and Astronomy, California Institute of Technology, Pasadena, CA 91125, USA; mhankins@astro.caltech.edu \\ ${ }^{2}$ Institute of Space \& Astronautical Science, Japan Aerospace Exploration Agency, 3-1-1 Yoshinodai, Chuo-ku, Sagamihara, Kanagawa 252-5210, Japan \\ ${ }^{3}$ SOFIA-USRA, NASA Ames Research Center, MS 232-12, Moffett Field, CA 94035, USA \\ ${ }^{4}$ SETI Institute, 189 Bernardo Ave., Mountain View, CA 94043, USA \\ ${ }^{5}$ Dept. of Physics and Astronomy, University of California, Los Angeles, CA 90095-1547, USA \\ ${ }^{6}$ Department of Physics and Astronomy, University of Kansas, 1251 Wescoe Hall Dr., Lawrence, KS 66045, USA \\ ${ }^{7}$ National Astronomical Observatory of Japan, 2-21-1 Osawa, Mitaka, Tokyo, 181-8588, Japan \\ ${ }^{8}$ Joint ALMA Observatory, Alonso de Córdova 3107, Vitacura, Santiago, Chile \\ ${ }_{9}^{9}$ Argelander-Institut für Astronomie, Universität Bonn, Auf dem Hügel 71, D-53121, Bonn, Germany \\ ${ }^{10}$ Department of Astronomy, Cornell University, Space Sciences Bldg, Ithaca, NY 14853-6801, USA \\ ${ }_{11}$ Astrophysics Research Institute, Liverpool John Moores University, 146 Brownlow Hill, Liverpool L3 5RF, UK \\ ${ }^{12}$ Department of Astrophysical and Planetary Sciences, University of Colorado, 389 UCB, Boulder, CO 80309, USA \\ ${ }^{13}$ Institut für Astro- und Teilchenphysik, Universität Innsbruck, Technikerstr. 25, A-6020 Innsbruck, Austria \\ ${ }^{14}$ European Space Agency, 3700 San Martin Drive, Baltimore, MD 21218, USA \\ Received 2020 January 5; revised 2020 February 28; accepted 2020 March 2; published 2020 May 5
}

\begin{abstract}
The Galactic Center contains some of the most extreme conditions for star formation in our Galaxy, as well as many other phenomena that are unique to this region. Given our relative proximity to the Galactic Center, we are able to study details of physical processes to a level that is simply not yet possible for more distant galaxies, yielding an otherwise inaccessible view of the nuclear region of a galaxy. We recently carried out a targeted imaging survey of mid-infrared bright portions of the Galactic Center at 25 and $37 \mu \mathrm{m}$ using the FORCAST instrument on the Stratospheric Observatory for Infrared Astronomy (SOFIA). This survey was one of the inaugural Legacy Programs from SOFIA cycle 7, observing a total area of $403 \operatorname{arcmin}^{2}\left(2180 \mathrm{pc}^{2}\right)$, including the Sgr A, B, and C complexes. Here we present an overview of the survey strategy, observations, and data reduction as an accompaniment to the initial public release of the survey data. We discuss interesting regions and features within the data, including extended features near the circumnuclear disk, structures in the Arched Filaments and Sickle H II regions, and signs of embedded star formation in Sgr B2 and Sgr C. We also feature a handful of less well studied mid-infrared sources located between Sgr A and Sgr C that could be sites of relatively isolated star formation activity. Last, we discuss plans for subsequent publications and future data releases from the survey.
\end{abstract}

Unified Astronomy Thesaurus concepts: H II regions (694); Galactic center (565); Star formation (1569); Star forming regions (1565)

\section{Introduction}

The environment in the Galactic Center (GC) is unlike any other part of our Galaxy. The region contains high molecular gas densities (Guesten \& Henkel 1983), high temperatures (Morris et al. 1983; Guesten et al. 1985), and large turbulent motions (Bally et al. 1987), all inside a deep gravitational potential well (Morris \& Serabyn 1996). The conditions in the GC mirror those found in the nuclei of luminous infrared galaxies and high-redshift systems near the peak of cosmic star formation history (Kruijssen \& Longmore 2013), but its relative proximity ( $d=8.0 \pm 0.5 \mathrm{kpc}$; Reid 1993) enables us to study the physical processes there at a level of detail that is simply not possible in more distant systems (see the recent review by Mills 2017).

Observations of the GC often challenge theoretical models of star formation, which often break down in this complex region. For example, while the GC comprises less than $0.01 \%$ of the total volume of the Galactic disk, its star formation rate (SFR; $\sim 0.1 M_{\odot} \mathrm{yr}^{-1}$; Immer et al. 2012; Barnes et al. 2017) is a considerable fraction of the total SFR of the Galaxy $\left(\sim 1.2 M_{\odot} \mathrm{yr}^{-1}\right.$; Lee et al. 2012). However, the global GC
SFR is more than an order of magnitude smaller than one would expect based on scaling relations with its dense molecular gas content (Lada et al. 2012; Longmore et al. 2013). The inefficiency of the $\mathrm{GC}$ in converting dense gas to stars presents a significant quandary for the region with much broader implications. For example, SFR measurements are universally used as a fundamental diagnostic tool for understanding the underlying physics in galaxies and for understanding galaxy evolution.

Even with the aforementioned star formation deficiency, the stellar inventory of the GC is relatively rich with numerous types of massive, evolved stars, such as luminous blue variables and Wolf-Rayet stars (e.g., Figer 2009). The GC is home to three known massive stellar clusters (Lu 2018): the Arches cluster (Cotera et al. 1996), the Quintuplet cluster (Nagata et al. 1990; Okuda et al. 1990), and the Central cluster (Krabbe et al. 1991, 1995), in addition to a large number of massive field stars that are spread throughout the region (e.g., Muno et al. 2006; Mauerhan et al. 2010; Dong et al. 2012). The origin of these massive field stars is somewhat of a mystery. While several sources may be former cluster members that have been dynamically ejected or removed by tidal 
evaporation, the entire population of these sources cannot be accounted for with these mechanisms (Habibi et al. 2014). Instead, some fraction of the field stars likely originate from a more isolated mode of star formation, and there is evidence of ongoing massive star and stellar cluster formation within parts of the GC (e.g., Barnes et al. 2019, and references therein). There have also been efforts to identify possible clusters associated with GC field stars, which have proven observationally challenging (Steinke et al. 2016; Dong et al. 2017), though there has been recent progress in this area using large proper motion studies (Shahzamanian et al. 2019).

Large extinction toward the GC $\left(A_{V} \sim 30\right.$; Fritz et al. 2011) makes it impossible to observe massive stars and protostars at optical and ultraviolet wavelengths. Instead, studies of the region have relied on observations at other wavelengths to examine the distribution and birth environment of stars. Numerous studies of star formation in the GC have been conducted with mid-infrared observations between $\sim 3.6$ and $24 \mu \mathrm{m}$ (e.g., Ramírez et al. 2008; Yusef-Zadeh et al. 2010; An et al. 2011; Immer et al. 2012). In particular, warm dust emission at $\sim 24 \mu \mathrm{m}$ is a valuable probe for identifying young stellar objects (YSOs) and estimating SFRs (e.g., Calzetti et al. 2007). However, the most active regions within the inner $\sim 200$ pc of our Galaxy are strongly saturated in the Spitzer/ MIPS $24 \mu \mathrm{m}$ data (Yusef-Zadeh et al. 2009). Earlier observations with the Midcourse Space Experiment (MSX; Egan et al. 2003 ) at $21.3 \mu \mathrm{m}$ (Band E) provide unsaturated images but are relatively low in spatial resolution $\left(\sim 20^{\prime \prime}\right.$ or $\left.\sim 0.8 \mathrm{pc}\right)$ when compared with Spitzer/MIPS at $24 \mu \mathrm{m}\left(\sim 6^{\prime \prime}\right.$ or $\left.\sim 0.2 \mathrm{pc}\right)$. The MSX data suffer from significant confusion in these complex regions, which presents a considerable hurdle in our understanding of these very active portions within the GC. Furthermore, the lack of high-quality mid-infrared data in these regions represents an essential missing piece of the rich multiwavelength picture of the GC that has emerged over the last decade.

In order to create improved mid-infrared maps of the brightest portions of the inner $\sim 200 \mathrm{pc}$ of our Galaxy, we set out to conduct a targeted survey of regions within the GC using the Stratospheric Observatory for Infrared Astronomy (SOFIA)/FORCAST. This survey was selected as one of the inaugural Legacy Programs in SOFIA cycle 7. Observations were obtained at 25 and $37 \mu \mathrm{m}$ using the Faint Object infraRed CAmera for the SOFIA Telescope (FORCAST), enabling us to create a high-resolution (FWHM $\sim 2$ ". 3 or $\sim 0.07$ pc at $25 \mu \mathrm{m}$ and FWHM $\sim 3$ ". 4 or $\sim 0.1 \mathrm{pc}$ at $37 \mu \mathrm{m})$ mosaic of portions within the GC including the Sgr A complex and other prominent star-forming regions such as Sgr B and Sgr C.

In this paper, we present a description of the observations along with the survey strategy and an initial look at the data set with various regions of interest highlighted. In Section 2, we provide details on the observations and information regarding the creation of the mosaics presented in this work. In Section 3, we highlight and discuss regions and sources that stand out as particularly interesting. These overviews are not intended to be a full and complete analysis of the objects discussed but rather to emphasize areas where this data set is providing an enhanced view of regions within the GC. We are planning follow-up papers for several of these features, as discussed in the text below. Finally, we provide a summary and future outlook in Section 4.

\section{Observations and Data Reduction}

Observations of our mid-infrared GC survey were carried out with the $2.5 \mathrm{~m}$ telescope aboard SOFIA (Young et al. 2012) using the FORCAST instrument (Herter et al. 2012). FORCAST is a $256 \times 256$ pixel dual-channel, wide-field mid-infrared camera with a field of view (FOV) of $3 ! 4 \times 3 ! 2$ and a plate scale of 0 ". 768 per pixel. The two channels consist of a short-wavelength camera (SWC) operating at 5-25 $\mu \mathrm{m}$ and a long-wavelength camera (LWC) operating at 28-40 $\mu \mathrm{m}$, and the instrument is capable of observing with both cameras simultaneously using a dichroic beam splitter.

\subsection{SOFIA/FORCAST Survey Plan}

The SOFIA/FORCAST Galactic Center survey was designed to map out infrared-bright regions within the inner $\sim 200$ pc of the Galaxy. We chose to conduct the survey using the FORCAST $25 \mu \mathrm{m}$ filter because it is close in wavelength to the Spitzer/MIPS $24 \mu \mathrm{m}$ filter and only a slight color correction ([24]-[25] $=0.15 \mathrm{mag}$ for a Vega-like source) is needed to compare photometry between the two data sets where possible. The FORCAST and MIPS data sets are complementary because the higher bright source limit of FORCAST allows for observations of objects and regions where the MIPS data are saturated, while the higher sensitivity of the MIPS probes fainter sources than FORCAST can detect in reasonable integration times. Our nominal sensitivity per field was selected to achieve a $5 \sigma$ point source depth of $250 \mathrm{mJy}$ at $25 \mu \mathrm{m}$, which is equivalent to a $3 \sigma$ extended source depth of $1200 \mathrm{MJy} \mathrm{sr}^{-1}$. This imaging depth allows for detection of class I YSOs (age $<1 \mathrm{Myr}$ ) down to a mass of $\sim 6 M_{\odot}$ based on the mean $24 \mu \mathrm{m}$ flux of YSO models from Robitaille et al. (2006). This imaging depth is also comfortably below the MIPS hard saturation limit for the existing $24 \mu \mathrm{m}$ GC map ( $\sim 400 \mathrm{mJy}$ for point sources or $\sim 2300 \mathrm{MJy} \mathrm{sr}^{-1}$ for extended emission), allowing for comparison of common sources between the MIPS and FORCAST surveys.

In addition to the $25 \mu \mathrm{m}$ filter, we used FORCAST's internal dichroic beam splitter to enable simultaneous observation with the long-wavelength camera, which results in only modest loss in the short-wavelength channel. ${ }^{15}$ To give the greatest wavelength coverage, we selected the longest wavelength filter available on FORCAST $(37.1 \mu \mathrm{m})$ to be paired with the $25 \mu \mathrm{m}$ filter observations.

Based on the integration times determined by the $25 \mu \mathrm{m}$ observations, the corresponding $37 \mu \mathrm{m} 5 \sigma$ point source depth was $550 \mathrm{mJy}$, and the equivalent $3 \sigma$ extended source depth was $2200 \mathrm{MJy} \mathrm{sr}^{-1}$. The nominal spatial resolutions of the 25 and $37 \mu \mathrm{m}$ filters in this observing mode are 2!"3 and 3.!4, respectively. ${ }^{16}$

Field pointings for the survey were planned using the MSX band E data (Figure 1). Additionally, we compared the survey footprint with the aforementioned $24 \mu \mathrm{m}$ Spitzer/MIPS data in order to optimize coverage of the hard saturated sources. In total, our survey plan covered more than $99 \%$ of the hard saturated area in the innermost $200 \mathrm{pc}$ of the Galaxy (Figure 1).

\footnotetext{
15 Throughput of the dichroic for the SWC from 11 to $25 \mu \mathrm{m}$ is $85 \%$, while the throughput of the LWC from 25 to $40 \mu \mathrm{m}$ is only $40 \%$.

16 https://www.sofia.usra.edu/science/proposing-and-observing/observershandbook-cycle-7/5-forcast
} 


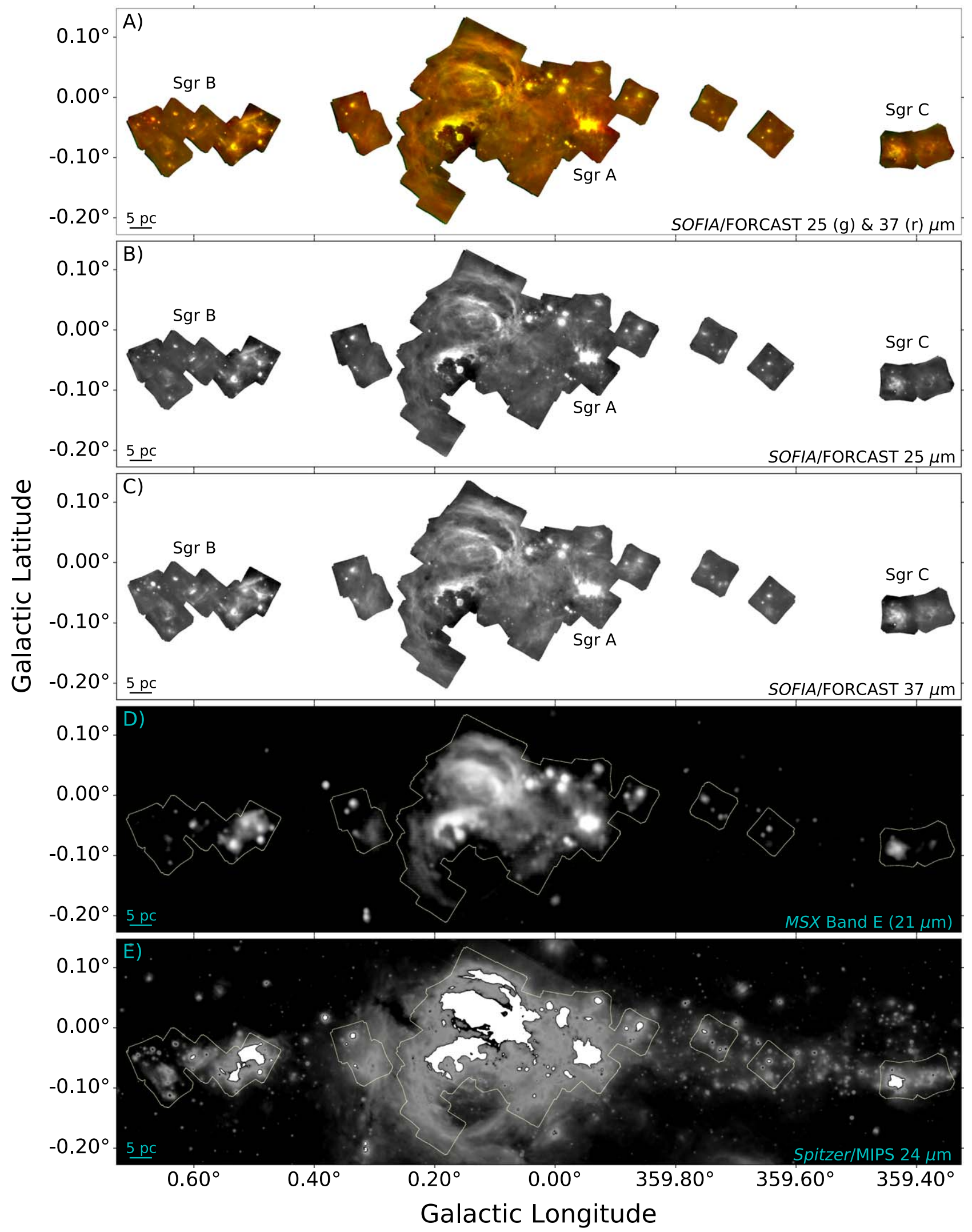

Figure 1. (A) SOFIA/FORCAST GC survey mosaic created using the 25.2 (green) and 37.1 (red) $\mu \mathrm{m}$ maps. Well-known regions including Sgr A, B, and C are labeled here and in panels (B) and (C). (B) The $25.2 \mu \mathrm{m}$ SOFIA/FORCAST survey mosaic shown in grayscale. (C) The $37.1 \mu \mathrm{m}$ SOFIA/FORCAST survey mosaic shown in grayscale. (D) MSX band E $(21 \mu \mathrm{m})$ data that were used for planning the FORCAST observations. The stretch of the data shows the approximate depth of the FORCAST observations for comparison and the footprint of the survey area. (E) The $24 \mu \mathrm{m}$ Spitzer/MIPS mosaic of the GC. Several of the brightest infrared features are hard saturated (shown in white). There are additional quality issues with portions of the map neighboring extended hard saturated regions, which have high backgrounds and suffer from other bright source effects (see Hinz et al. 2009). The stretch of this figure demonstrates the relative depth of the MIPS mosaic and shows numerous sources outside of the FORCAST survey footprint that have useful data. 
Table 1

SOFIA/FORCAST Observation Details

\begin{tabular}{|c|c|c|c|c|c|c|c|c|}
\hline Field ID & Field Center $(l, b)$ & Sky Angle $\left({ }^{\circ}\right)^{a}$ & $25 \mu \mathrm{m} t_{\text {int }}(\mathrm{s})$ & $37 \mu \mathrm{m} t_{\text {int }}(\mathrm{s})$ & Chop Angle $\left({ }^{\circ}\right)^{b}$ & Chop Throw (") & Comment $^{c}$ & AOR ID \\
\hline 1 & $(359.376,-0.080)$ & 148.3 & 402 & 369 & 88 & 140 & Affected & $\overline{07 \_0189 \_1}$ \\
\hline 2 & $(359.429,-0.087)$ & 130.1 & 385 & 353 & 52 & 115 & Affected & 07_0189_2 \\
\hline 3 & $(359.641,-0.062)$ & 83.5 & 404 & 315 & 60 & 160 & Post-fix & 07_0189_3 \\
\hline 5 & $(359.737,-0.018)$ & 99.7 & 461 & 360 & 308 & 165 & Post-fix & 07_0189_5 \\
\hline 6 & $(0.310,-0.058)$ & 153.1 & 458 & 415 & 110 & 135 & Post-fix & 07_0189_6 \\
\hline 7 & $(0.337,-0.024)$ & 138.9 & 217 & 192 & 230 & 130 & Post-fix & 07_0189_7 \\
\hline 8 & $(0.584,-0.053)$ & 174.3 & 433 & 404 & 200 & 190 & Affected & 07_0189_8 \\
\hline 9 & $(0.631,-0.041)$ & 178.2 & 614 & 626 & 220 & 160 & Nominal & 07_0189_9 \\
\hline 10 & $(0.641,-0.092)$ & 165.1 & 528 & 480 & 77 & 200 & Affected & 07_0189_10 \\
\hline 11 & $(0.674,-0.051)$ & 146.2 & 210 & 176 & 130 & 210 & Affected & 07_0189_11 \\
\hline 12 & $(0.534,-0.072)$ & 163.3 & 414 & 387 & 80 & 160 & Affected & 07_0189_35 \\
\hline 13 & $(0.496,-0.051)$ & 184.7 & 522 & 532 & 60 & 160 & Nominal & 07_0189_36 \\
\hline A & $(359.867,-0.007)$ & 100.0 & 478 & 400 & 240 & 175 & Affected & 07_0189_12 \\
\hline B & $(359.942,0.027)$ & 130.2 & 486 & 454 & 270 & 160 & Affected & 07_0189_13 \\
\hline $\mathrm{C}$ & $(359.931,-0.019)$ & 85.6 & 440 & 368 & 265 & 210 & Affected & 07_0189_14 \\
\hline $\mathrm{D}$ & $(359.934,-0.067)$ & 88.3 & 458 & 415 & 5 & 140 & Affected & 07_0189_15 \\
\hline E & $(359.970,-0.019)$ & 143.6 & 393 & 319 & 245 & 210 & Affected & 07_0189_16 \\
\hline $\mathrm{F}$ & $(359.975,-0.064)$ & 165.5 & 472 & 433 & 352 & 190 & Affected & 07_0189_17 \\
\hline G & $(0.015,-0.022)$ & 161.0 & 492 & 399 & 250 & 205 & Affected & 07_0189_18 \\
\hline $\mathrm{H}$ & $(0.014,-0.078)$ & 188.5 & 527 & 590 & 28 & 200 & Affected & 07_0189_19 \\
\hline I & $(0.040,-0.124)$ & 183.4 & 505 & 458 & 45 & 180 & Affected & 07_0189_20 \\
\hline K & $(0.051,-0.008)$ & 172.3 & 505 & 397 & 260 & 200 & Affected & 07_0189_22 \\
\hline $\mathrm{L}$ & $(0.056,-0.053)$ & 183.4 & 524 & 451 & 98 & 210 & Affected & 07_0189_23 \\
\hline M & $(0.065,-0.089)$ & 164.8 & 493 & 447 & 118 & 173 & Post-fix & 07_0189_24 \\
\hline $\mathrm{O}$ & $(0.101,-0.023)$ & 181.0 & 472 & 451 & 78 & 210 & Affected & 07_0189_26 \\
\hline$P$ & $(0.102,-0.071)$ & 136.4 & 525 & 511 & 95 & 145 & Nominal & 07_0189_27 \\
\hline Q & $(0.145,0.006)$ & 188.0 & 518 & 500 & 190 & 210 & Affected & 07_0189_28 \\
\hline $\mathrm{R}$ & $(0.200,0.023)$ & 174.9 & 427 & 387 & 225 & 210 & Affected & 07_0189_29 \\
\hline S & $(0.182,-0.018)$ & 176.6 & 469 & 430 & 180 & 200 & Affected & 07_0189_30 \\
\hline $\mathrm{T}$ & $(0.223,-0.043)$ & 159.2 & 492 & 479 & 205 & 200 & Affected & 07_0189_31 \\
\hline $\mathrm{U}$ & $(0.223,-0.089)$ & 148.4 & 468 & 437 & 82 & 210 & Affected & 07_0189_32 \\
\hline V & $(0.216,-0.134)$ & 175.4 & 505 & 458 & 75 & 140 & Post-fix & 07_0189_33 \\
\hline $\mathrm{W}$ & $(0.190,-0.169)$ & 180.9 & 482 & 500 & 80 & 140 & Post-fix & 07_0189_34 \\
\hline $\mathrm{X}$ & $(0.142,-0.045)$ & 181.6 & 484 & 451 & 60 & 180 & Post-fix & 07_0189_37 \\
\hline $\mathrm{Y}$ & $(0.175,-0.067)$ & 170.6 & 504 & 471 & 35 & 150 & Post-fix & 07_0189_38 \\
\hline
\end{tabular}

Notes.

${ }^{\text {a }}$ Sky angle refers to the orientation of the field measured in degrees east of celestial north. All angles in this table are measured with respect to the celestial frame rather than the galactic frame to be consistent with the convention of the data provided by the observatory.

${ }^{\mathrm{b}}$ Chop angle refers to the direction of the chop throw, which is also the direction of PSF elongation in the fields affected by the secondary mirror issue described in the text.

${ }^{c}$ Data quality comments refer to the chopper mirror issue described in Section 2.2. Data labeled "Affected" have noticeable PSF elongation in the direction of the Chop Angle. Data labeled "Nominal" or "Post-fix" are unaffected by the mirror issue.

\subsection{SOFIA/FORCAST Cycle 7 Observations}

A total of 35 fields were observed during SOFIA cycle 7 as part the FORCAST Galactic Center Legacy Program (Program ID: 07-0189; PI: Hankins). Here, we provide a brief description of the observations, with further details provided in Table 1. All 35 fields were observed during the annual SOFIA southern hemisphere deployment to Christchurch, NZ. Observations for the program were spread over eight total flights that occurred between 2019 July 1 and 11.

Chopping and nodding was used to remove the sky and telescope thermal backgrounds for all observations. This technique requires off fields that are devoid of emission to properly subtract the background emission. The GC is a particularly complex emitting environment, and there were instances where we did not have a perfectly blank sky to nod and chop onto within telescope limits. In these cases, we selected the "best" available off field that minimized the number of sources (most frequently an individual source within the expected detection limits). We employed the $\mathrm{C} 2 \mathrm{NC} 2$ observing mode to allow use of the full FORCAST FOV, even though this mode comes with considerable overheads, due to the necessary three off-source positions. Dithering was used to remove bad pixels and mitigate response variations. We employed different dither patterns for several of the fields in order to minimize observing inefficiencies related to the C2NC2 observing mode, which does not adversely impact the data quality between fields.

On average, we achieved $462 \mathrm{~s}$ of integration for each field in the $25 \mu \mathrm{m}$ filter and an average of $423 \mathrm{~s}$ for the $37 \mu \mathrm{m}$ filter. A listing of integration times for each field individually can be found in Table 1. The estimated total photometric errors for the 25 and $37 \mu \mathrm{m}$ data are $\sim 10 \%$. Two fields (7 and 11) have integration times that are substantially below the average and were cut short by scheduling issues or adjustments that were needed in flight. However, the depth of these images is still 
suitable for some science goals (corresponding to a $250 \mathrm{mJy}$ point-source detection of $\sim 3.7 \sigma$ rather than $5 \sigma$ ). In addition to these fields with low signal-to-noise ratio $(\mathrm{S} / \mathrm{N})$, three additional fields were planned as part of the survey but were not observed in cycle 7 because of time and observability constraints.

During the flight series, there was an issue with the secondary chopper mirror on the telescope that caused sources to be elongated in the direction of the chop throw. Consequently, in Table 1 we have included information regarding the extent to which each field was affected by the secondary mirror issue. Fields where the elongation is known to be an issue are labeled "affected." Fields that may also have been impacted, but only slightly, are labeled "nominal," while those taken after the issue was corrected are labeled "post-fix." In the affected data, pointspread function (PSF) ellipticities range between $\sim 0.1$ and 0.4; however, several of the fields contain few and in some cases no suitable PSF reference stars, which makes measuring the data quality effects challenging. Using a deconvolution method with a suitable PSF model should be able to correct the PSF variation to an extent, although this is a complex process given the level of PSF variation in the "affected" fields. We are planning to produce a set of corrected maps with a more uniform PSF to be made public with a future data release.

\subsection{SOFIA/FORCAST Observations from Prior Cycles}

In addition to the observations carried out in SOFIA cycle 7, suitable data from earlier cycles were considered for inclusion in our GC survey. Several guaranteed time observations with FORCAST were focused on targets within the GC, including the Circumnuclear Ring (Lau et al. 2013), the Sickle H II region (Lau et al. 2014a; Hankins et al. 2016), and the Sgr A East H II regions (Lau et al. 2014b).

For the survey mosaic presented in this work, we only included observations taken in SOFIA cycle 3 or later, which consists of the Arched Filaments H II region (Hankins et al. 2017) and the H H II regions (Hankins et al. 2019). Observation details for these data can be found in the above-referenced works, and a description of their incorporation in our survey mosaic can be found in the following subsection.

\subsection{Data Processing}

Observations were processed using the pipeline steps described in Herter et al. (2013). The Level 3 processed data products were downloaded from the SOFIA Data Cycle System (DCS). ${ }^{17}$ Images from each individual pointing were mosaicked using the SOFIA Data Pipeline software REDUX (Clarke et al. 2015) in order to construct the preliminary FORCAST Level 4 imaging mosaics that are presented in this work. Both the Level 3 and 4 data products from this program are available for download via the SOFIA DCS and the NASA/IPAC Infrared Science Archive (IRSA). ${ }^{18}$

Creating the mosaic for this data set resulted in several challenges that were addressed as follows. First, observations using FORCAST on bright sources cause a negative signal offset throughout the detector that lowers the background flux levels. To correct for this, background levels were subtracted on a field-by-field basis to bring the overall background values

\footnotetext{
17 https://dcs.arc.nasa.gov

18 https://irsa.ipac.caltech.edu/frontpage/
}

Table 2

Known Data Artifacts Removed in Postprocessing

\begin{tabular}{lccc}
\hline \hline Location $(l, b)$ & Filter(s) & Type $^{\mathrm{a}}$ & Comment $^{\mathrm{b}}$ \\
\hline$(0.103,-0.086)$ & Both & point & \\
$(0.098,-0.079)$ & Both & point & \\
$(0.090,-0.070)$ & Both & point & \\
$(359.933,0.024)$ & $25 \mu \mathrm{m}$ & point & dithered \\
$(359.894,-0.008)$ & $25 \mu \mathrm{m}$ & point & dithered \\
$(359.896,-0.012)$ & $25 \mu \mathrm{m}$ & point & dithered \\
$(359.892,-0.015)$ & $25 \mu \mathrm{m}$ & point & \\
$(0.053,-0.086)$ & $25 \mu \mathrm{m}$ & point & \\
$(0.098,0.055)$ & $25 \mu \mathrm{m}$ & point & \\
$(0.103,0.074)$ & $25 \mu \mathrm{m}$ & point & \\
$(0.126,0.111)$ & Both & point & nearby source \\
$(0.115,0.081)$ & $25 \mu \mathrm{m}$ & point & \\
$(0.482,-0.077)$ & Both & point & \\
\hline
\end{tabular}

Notes.

a Specifies if the artifact is point-like or extended.

b Comments about image artifacts. The "dithered" artifact is the same source appearing in multiple locations due to the dither pattern. The "nearby source" label denotes a nearby positive source where the photometry may be impacted by the artifact.

to approximately zero. Next, as previously discussed, chopping and nodding require off fields that are free of detectable mid-IR emission in order to properly subtract sky and telescope backgrounds. In cases where off fields contained sources, this results in negative point sources or regions in the data. To correct for these negative artifacts, we modeled point sources using a 2D Gaussian, and if they achieved sufficient $S / N(\gtrsim 5)$, the source model was subtracted from the data, leaving behind the fitted background at the location of the artifact. A summary of the locations of removed sources can be found in Table 2.

Next, the issue that required the most effort to correct involved astrometry. Telescope pointing with FORCAST is only accurate to within a few pixels ( $\sim \operatorname{arcseconds),~so~}$ astrometry was absolutely calibrated using the available Spitzer and MSX data by matching up the centroids of point sources in common between those maps and the SOFIA data. Precise alignment was difficult in the case of aligning to the lowerresolution MSX $21 \mu \mathrm{m}$ data or the saturated regions of the Spitzer/MIPS $24 \mu \mathrm{m}$ data. However, a handful of bright $8 \mu \mathrm{m}$ point sources were also detected at $25 \mu \mathrm{m}$ and aided in the alignment of several of the fields.

Slight changes in the focal plane distortion across the array and limited calibration data also contributed to some elongation and smearing of sources on scales similar to the elongation caused by the secondary chopping mirror issue as discussed above. This distortion is variable across the array, with the worst effects at the edges resulting in some misalignment of up to a few pixels ( $\sim$ arcseconds). Based on these issues, we estimate the astrometry of the final SOFIA mosaic is at worst three pixels or $\sim 2^{\prime \prime}$. However, comparing common sources between FORCAST and Spitzer suggests the FORCAST mosaic astrometry is typically better than one pixel. ${ }^{19}$ More calibration data are expected to be taken in the next SOFIA cycle, in which case the overall astrometric precision of the maps may be improved in a future data release.

\footnotetext{
19 The median offset for a modest-sized sample $(N=25)$ of randomly selected sources between the data sets is $\sim 0.4$ pixels or $\sim 0$." 3 .
} 


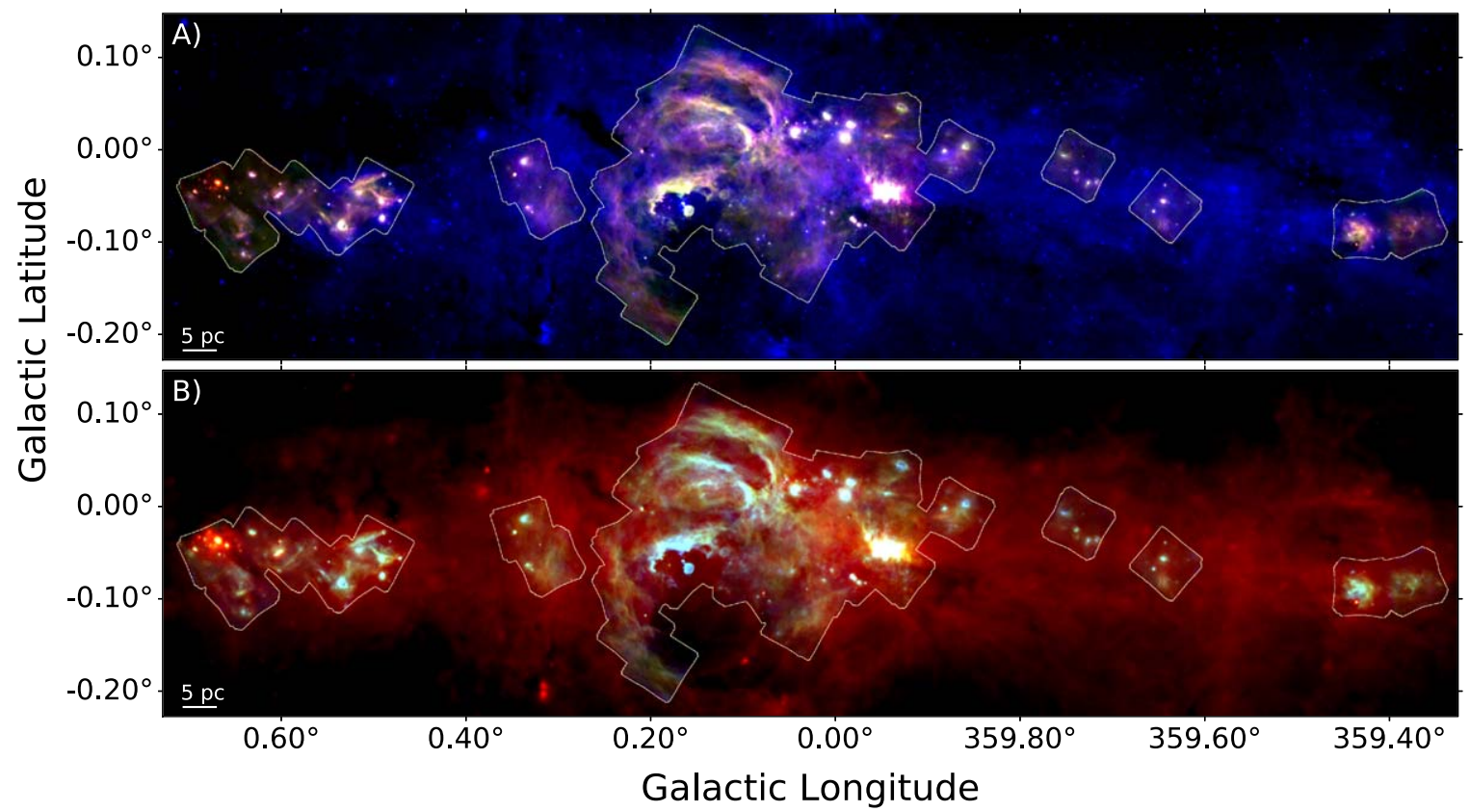

Figure 2. Comparison of the SOFIA/FORCAST mosaics with other infrared survey maps. (A) False-color map of the survey region using Spitzer/IRAC $8 \mu \mathrm{m}$ (blue), SOFIA/FORCAST $25 \mu \mathrm{m}$ (green), and $37 \mu \mathrm{m}$ (red). (B) Additional false-color map of the survey region using SOFIA/FORCAST $25 \mu \mathrm{m}$ (blue), $37 \mu \mathrm{m}$ (green), and Herschel/PACS $70 \mu \mathrm{m}$ (red). Both figures show an outline of the SOFIA/FORCAST survey footprint in white.

Finally, changes to the data reduction pipeline between SOFIA cycle 7 and earlier cycles presented several issues for combining the various data sets into the mosaics presented in this work. These issues were largely overcome by reprocessing the earlier cycle 3 and 4 data using REDUX with modifications to improve backward compatibility. While we present initial mosaics produced as part of this program, there is still work needed to optimally combine the older and newer data sets. For example, the new processing for the $37 \mu \mathrm{m}$ observations has resulted in a three-pixel-wide gap in a small section between two fields in the Arched Filaments near (0.116, 0.073), and there is a similar one-pixel-wide gap in a section between another set of fields in the $25 \mu \mathrm{m}$ map located near $(0.148$, $0.065)$. Neither of these gaps where present in earlier versions of the data at the time they were processed in cycles 3 and 4, and they may point to an issue with differences in distortion correction over time. For the present data release, we have used neighboring pixels to interpolate over these "missing" pixels in the small gap areas. As part of a future data release, we are planning updates to the REDUX package that will help with these and other issues in order to improve future versions of the survey mosaics.

\subsection{Additional Data from the Literature}

In our initial study of the SOFIA/FORCAST mosaics, we compared the maps with a number of other prominent GC surveys from the literature, including the Hubble Space Telescope (HST) Paschen- $\alpha$ Survey of the Galactic Center (Wang et al. 2010), the GLIMPSE Spitzer/IRAC survey (Churchwell et al. 2009), and the Herschel Hi-GAL survey (Molinari et al. 2010). These data sets provide a number of useful comparisons that we will discuss in subsequent sections. We also referenced maps and data from a number of other works in the literature that were focused on individual regions within the mosaics, and these are discussed in the relevant sections in the text.

\section{Discussion}

\subsection{Comparison with Other IR Surveys}

The GC is probably one of the most surveyed portions of the sky, and a number of high-quality data sets exist at nearly all available wavelengths. In this section, we focus on comparisons between the FORCAST mosaics and earlier survey maps produced by Spitzer/IRAC at $8 \mu \mathrm{m}$ and Herschel/PACS at $70 \mu \mathrm{m}$. Three-color combinations of these data sets can be found in Figure 2.

The morphology of the $8 \mu \mathrm{m}$ emission contains numerous similarities, but also important differences when compared to the 25 and $37 \mu \mathrm{m}$ emission. The origin of the $8 \mu \mathrm{m}$ emission differs from that at 25 and $37 \mu \mathrm{m} ; 8 \mu \mathrm{m}$ emission primarily traces very small, transiently heated grains, particularly polycyclic aromatic hydrocarbons (PAHs), which have prominent emission features in this wavelength region, while longer wavelength emission traces emission from larger, presumably silicate grains. Throughout the region, there is a diffuse $8 \mu \mathrm{m}$ component corresponding to most of the IR-bright regions at 25 and $37 \mu \mathrm{m}$, with the $8 \mu \mathrm{m}$ emission appearing more extended in comparison. There are notable exceptions to this trend, however, which likely points to variations in extinction over the survey area that can affect our observations even at longer wavelengths. For example, Sgr B2 is relatively dark at $8 \mu \mathrm{m}$ compared to many other regions within the survey, and sources in this region of the FORCAST data have very red colors. Both of these qualitative indicators suggest high extinction, and in the case of Sgr B2 it is already well established that this region suffers from significant local extinction (e.g., Scoville et al. 1975).

Comparison of the $70 \mu \mathrm{m}$ emission and the 25 and $37 \mu \mathrm{m}$ emission highlights a number of notable features. The emission at each of these wavelengths is primarily a thermal continuum, with the $25 \mu \mathrm{m}$ emission tracing relatively warm $\sim 100 \mathrm{~K}$ dust and the $70 \mu \mathrm{m}$ emission tracing cooler $\sim 40 \mathrm{~K}$ dust. Many of 


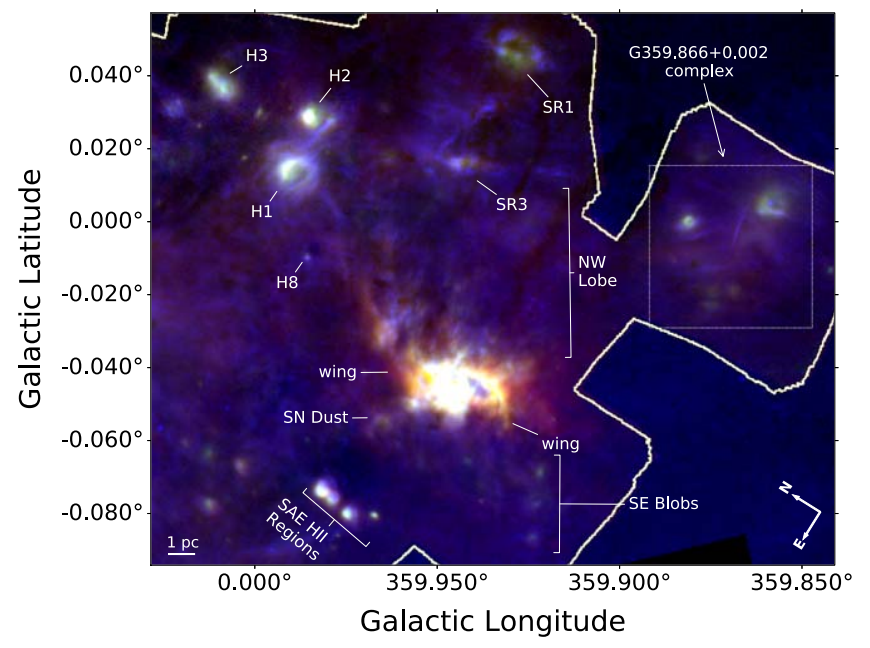

Figure 3. False-color map of the Sgr A complex created with the HST Paschen- $\alpha$ emission (blue), SOFIA/FORCAST $25 \mu \mathrm{m}$ (green), and $37 \mu \mathrm{m}$ (red) data. Several of the features discussed in the text are labeled for reference. Sgr A East is abbreviated "SAE" for brevity in the figure label. The dashed box shows the region around the G359.866+0.002 complex, which is featured in Figure 4, and an outline of the FORCAST survey footprint is also overlaid as a solid white outline.

the $\mathrm{H}$ II regions throughout the GC contain significant emission from warm dust, which results in bright emission at $25 \mu \mathrm{m}$ (Figure 2). This highlights the importance of the $25 \mu \mathrm{m}$ data when studying recent star formation both in giant $\mathrm{H}$ II regions like the Arched Filaments and Sickle and in smaller H II regions that may be associated with individual massive stars. Furthermore, the spatial resolution of FORCAST enables us to resolve many "bubble-like" structures down to a physical scale of $\sim 0.1 \mathrm{pc}$. For isolated sources, this is sufficient to resolve all but the most compact $\mathrm{H}$ II regions at the GC distance.

Another striking feature of the 25, 37, and $70 \mu \mathrm{m}$ data in Figure 2 is the number of compact red sources spread throughout the region. Prime examples of this can be seen in both Sgr B2 and Sgr C. In particular, Sgr B2 contains several of the most luminous $70 \mu \mathrm{m}$ sources in the GC (see Section 3.4). In the FORCAST data, we clearly observe these same objects with strong emission at $37 \mu \mathrm{m}$, suggesting deeply embedded star formation.

\subsection{Sgr A Complex}

One of the most interesting regions within the FORCAST mosaics is the Sgr A complex. Some of the earliest FORCAST observations of the GC were focused on the Circumnuclear Disk (CND; Lau et al. 2013); but the limited field of view prevented study of any large, extended structures that might be associated. Our survey significantly expands the coverage of this region, allowing us to examine various warm dust features extending from the position of the CND out to several parsecs. Among the highlights from this region is the mapping out of mid-infrared emission at the position of the NW X-ray lobe extending from the CND (Ponti et al. 2015). In addition, we observe several other extended structures that have been noted in radio and ionized gas observations of the region, enabling us to compare the warm dust and ionized gas throughout the region (e.g., Zhao et al. 2016).

We note remarkably detailed structures in the warm dust emission in this region (Figure 3). Outside of the brightest midIR features tracing the "minispiral" feature and the inner edge of the CND, also referred to as the Circumnuclear Ring (CNR), we observe fainter, extended structures protruding from the CNR that correspond to the well-known "wing" features (often referred to as the NW and SE Wings; Zhao et al. 2016). Similar streamers are also detected in this region in molecular gas (Liu et al. 2012), and the emission observed by FORCAST may be related to these molecular clouds. Dust near the NW wing appears to extend for several arcminutes, terminating near the position of the source $\mathrm{H} 1$ in the $\mathrm{H} \mathrm{H}$ II regions. This structure also appears to trace the edge of the NW X-ray lobe, although more detailed analysis is needed to understand possible interaction between the X-ray emitting region and the dusty infrared clouds.

At the position of the NW lobe, we find relatively faint, extended mid-IR emission that extends northward to a few relatively bright $25 \mu \mathrm{m}$ objects. These $25 \mu \mathrm{m}$ sources appear to be associated with the "smoke rings" discussed in Zhao et al. (2016). In the infrared, only one of these sources has a ring-like appearance (smoke ring \#1; SR1 in Figure 3) and might be associated with the nearby massive star CXOGC J174516.7 -285824 (Mauerhan et al. 2010). The IR emission of the third smoke ring (SR3) appears to "fill in" the ring-like structure observed in radio and ionized gas and suggests this feature may simply be a portion of a molecular cloud with prominent ionized edges. The second smoke-ring structure from Zhao et al. (2016) has little to no detectable emission in the midinfrared wavelengths presented in this work.

To the east of the CNR, we see the dust emission associated with the Sgr A East supernova remnant that was reported in Lau et al. (2015). To the south of the CND, there are a number of relatively faint sources and structures. The locations of several of these features appear to correspond to the locations of the "SE blobs" reported by Zhao et al. (2016) and may be related to disrupted cloudlets in the region (see Figure 7 from Liu et al. 2012). The origin of material in this region is of particular interest because it could signify outflow from the CND (e.g., Wang et al. 2010). We will explore these and other IR features in greater detail in a future paper focusing on Sgr A.

The H II region complex G359.866+0.002 (Figure 3) lies to the equatorial southwest of the CND with a projected separation of $\sim 2.5^{\prime}(\sim 6 \mathrm{pc})$. While this complex is not typically discussed in the context of Sgr A, this region contains a number of interesting features and is the southernmost portion of our coverage of Sgr A. G359.866+0.002 was found as a collection of Paschen- $\alpha$ emitting sources in Wang et al. (2010), where it was featured for its linear ionized gas features. In the FORCAST data, we observe dust emission at 25 and $37 \mu \mathrm{m}$ that traces many of these same narrow ionized gas features (Figure 4). These structures could point to locally strong magnetic fields or shocks that are sculpting the gas and dust emission. We also note two extended nebulae in the complex that are prominent at $25 \mu \mathrm{m}$, indicating dust that is likely being heated by luminous nearby stars. The $25 \mu \mathrm{m}$ nebulae G359.858 +0.004 may be associated with the nearby emission-line star 2MASS J17451618-2903156 from Mauerhan et al. (2010), while the other $25 \mu \mathrm{m}$ source $(\mathrm{G} 359.846+0.001)$ is cataloged as a YSO candidate based on ISOGAL data (Immer et al. 2012). However, the latter source is not present in the YSO catalog published in Yusef-Zadeh et al. (2009) and was not observed as part of the Spitzer/IRS sample of Galactic Center YSOs presented in An et al. (2011). To the south of these sources, there is a collection of dusty ridges visible at $37 \mu \mathrm{m}$, 

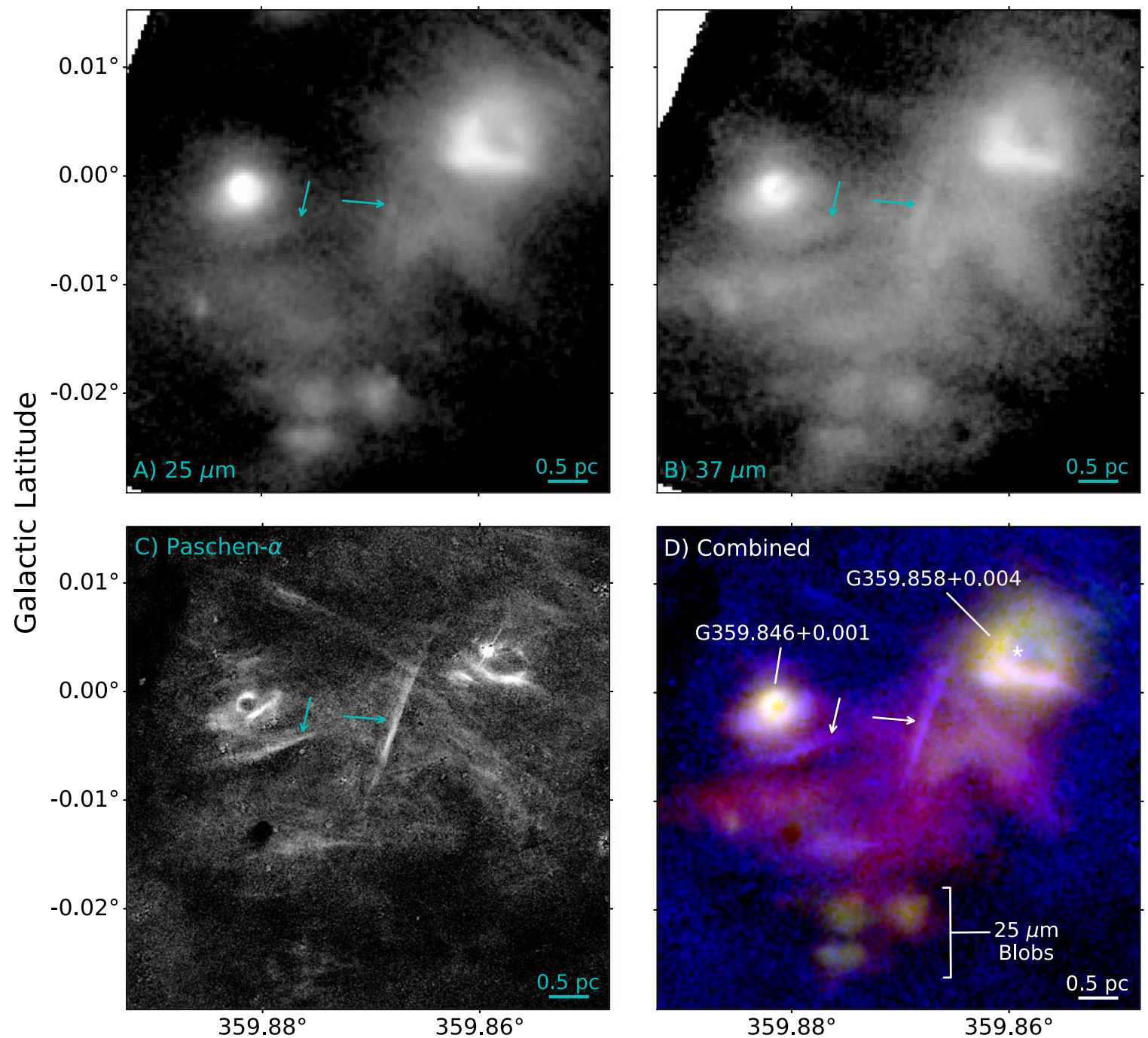

Galactic Longitude

Figure 4. (A) SOFIA/FORCAST $25 \mu \mathrm{m}$ data of the G359.866+0.002 complex. Two notable linear features are marked with arrows both here and in the following panels. (B) SOFIA/FORCAST $37 \mu \mathrm{m}$ data of the G359.866+0.002 complex. (C) HST Paschen- $\alpha$ data of the G359.866+0.002 complex. (D) False-color map of the G359.866+0.002 complex created with the Paschen- $\alpha$ (blue), $25 \mu \mathrm{m}$ continuum (green), and $37 \mu \mathrm{m}$ continuum (red) data. Additional labels are provided for the two dusty nebulae discussed in the text, and the location of the emission-line star 2MASS J17451618-2903156 is marked with a white star. Three extended sources to the south are labeled as the $25 \mu \mathrm{m}$ blobs.

some of which have associated ionized gas emission and others which do not. Further to the south, there is a collection of interesting extended $25 \mu \mathrm{m}$ sources, which we refer to as $25 \mu \mathrm{m}$ "blobs." As part of a future data release for this program, we will produce source catalogs for the 25 and $37 \mu \mathrm{m}$ data sets and publish additional information on possible YSO candidates determined from infrared colors of the SOFIA data.

\subsection{Arched Filaments and Sickle H II Regions}

The Arched Filaments and Sickle H II regions are some of the most prominent structures in the GC at both infrared and radio wavelengths (e.g., Yusef-Zadeh \& Morris 1987). Earlier work has been presented on SOFIA/FORCAST observations of both of these regions (e.g., Lau et al. 2014a, 2016; Hankins et al. 2016, 2017); however, our discussion of the present GC survey would be incomplete without some mention of these important regions. In this section, we highlight how the combined archival and new survey data can improve our picture of the physical processes at work in the Arched Filaments and Sickle (Figure 5).

The Arched Filaments complex is a collection of filamentary features that have a distinctive arched morphology due to our viewing geometry of the system (Lang et al. 2001, 2002). These structures are well known for their relation to the nonthermal emission in the GC Radio Arc, which meets with and undergoes a brightness discontinuity at the northern edge of the Arched Filaments (Lang et al. 2001) and appears to travel through the position of the Sickle H II region (Lang et al. 1997). Dust temperatures within the Arched Filaments H II region are consistent with the Arches cluster being the primary heating source (Hankins et al. 2017), as well as the primary source of ionizing radiation (e.g., Lang et al. 2001). Our previous FORCAST study of this region did not reveal any signs of ongoing star formation within the infrared-bright filament structures (Figure 5, upper panel; Hankins et al. 2017). Furthermore, we do not detect any infrared point sources near the location of the Arches cluster at 25 or $37 \mu \mathrm{m}$, which points 


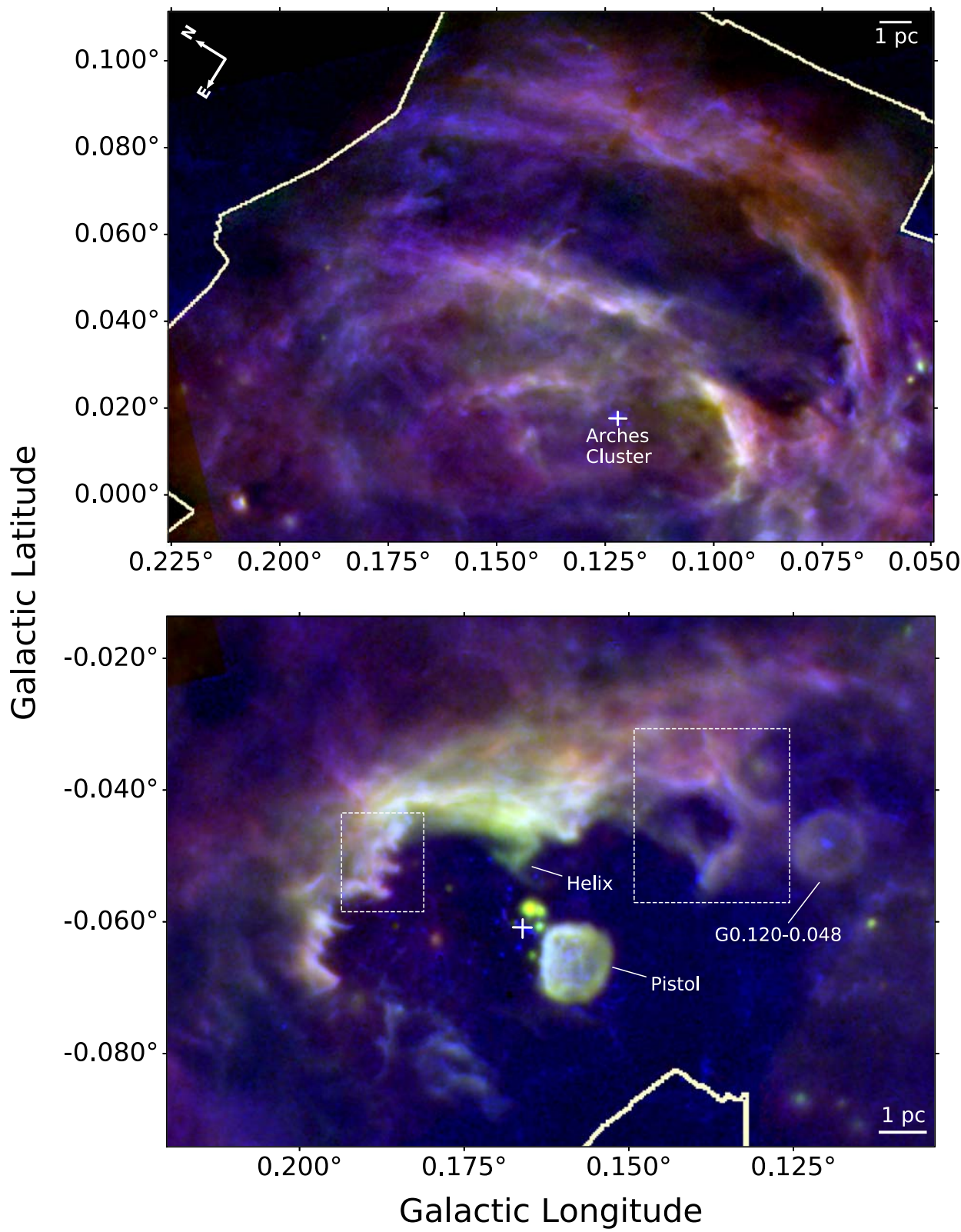

Figure 5. False-color map of the Arched Filaments (top) and the Sickle H II region (bottom) created with the HST Paschen- $\alpha$ emission (blue), SOFIA/FORCAST $25 \mu \mathrm{m}$ (green), and $37 \mu \mathrm{m}$ (red) data. The approximate locations of the Arches and Quintuplet clusters are marked with a white crosshair. Several infrared sources associated with the Quintuplet cluster can be seen near the marker, while the Arches cluster has a distinct lack of bright mid-infrared counterparts. Additional sources discussed in the text are labeled for reference, while the two dashed boxes indicate regions that are featured in a subsequent figure. An outline of the FORCAST survey footprint is also overlaid as a solid white outline in both plots. Previous FORCAST observations of these regions have been featured in earlier works (Hankins et al. 2016, 2017; Lau et al. 2016), which provide more detailed analysis.

to a lack of evolved, dust-forming stars, due to its relatively young age ( 2-3 Myr; Figer et al. 1999; Stolte et al. 2002).

The Sickle $\mathrm{H}$ II region has a number of interesting features as seen both in the morphology of the Sickle proper and in the numerous infrared sources primarily related to the Quintuplet cluster. First, the northern portion of the Sickle cloud (the "blade") shows several finger-like structures (Figure 5, lower panel, and Figure 6) reminiscent of features in M16 (Cotera et al. 2006). It is unclear if these structures are actively starforming like their M16 counterparts, although we may simply lack sufficient sensitivity and spatial resolution to adequately determine this at the GC distance. The morphological differences between the northern and western parts of the Sickle suggest they are influenced by the magnetic field organization within the cloud. Notably, the perpendicular part of the cloud (the "handle") does not display similar "fingers." However, there is a relatively faint, wispy, helical structure protruding from the "handle" that Lau et al. (2016) suggest is related to outflow from a massive star that appears near the cloud edge.

Gas and dust within the Sickle are most likely ionized and heated by the highly luminous Quintuplet cluster $(L=3 \times$ $10^{7} L_{\odot}$; Figer et al. 1999). Unlike the Arches cluster, the Quintuplet cluster contains five luminous infrared sources from which the cluster name is derived (Nagata et al. 1990; Okuda et al. 1990). These five "Quintuplet proper members" are likely Wolf-Rayet stars that are undergoing active dust production (Tuthill et al. 2006; Najarro et al. 2017), and they represent 


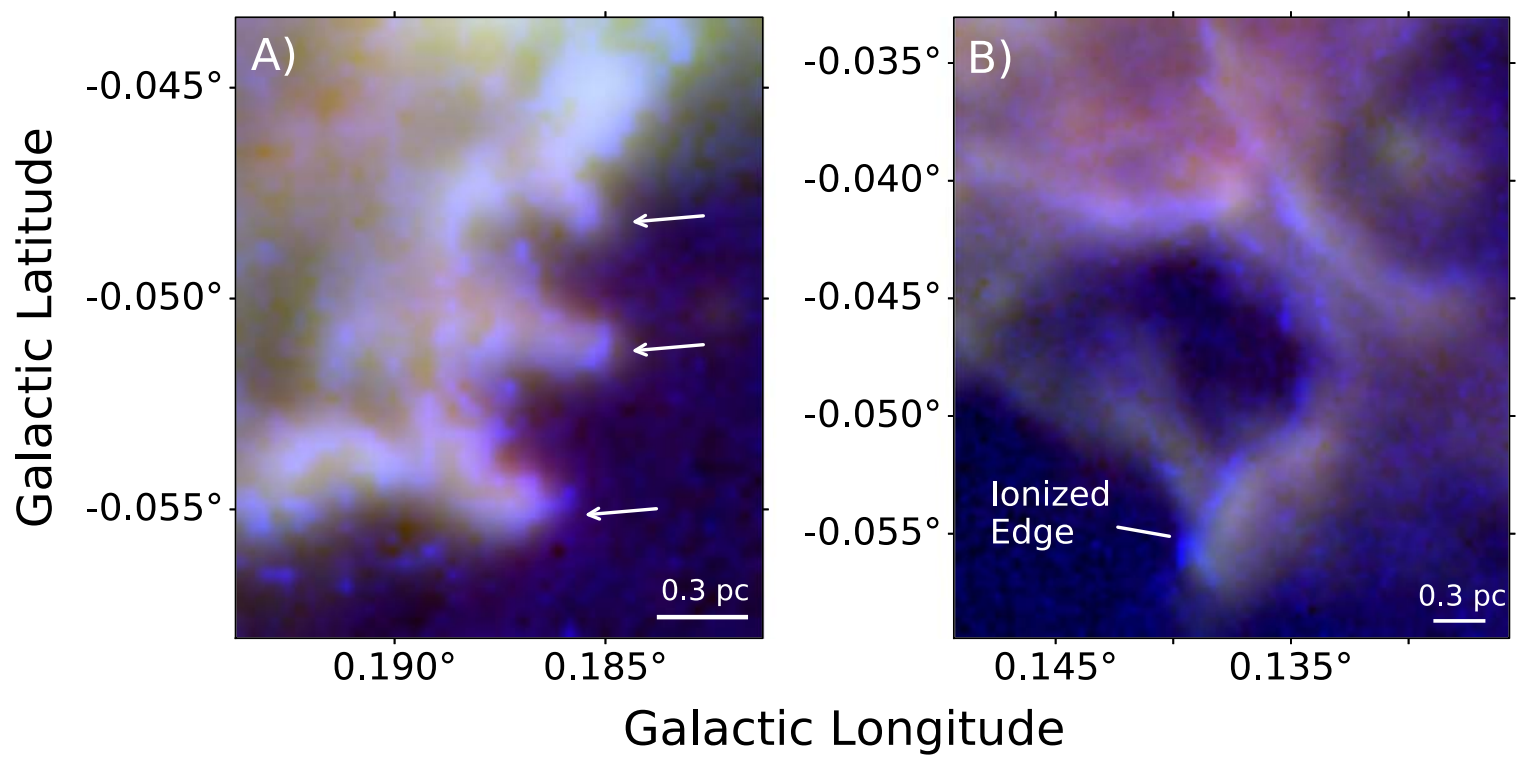

Figure 6. Zoom-in on two interesting regions within the Sickle H II region. Panel (A) shows several of the finger-like structures in the "blade" region. Arrows point out a few of the more prominent of these features that appear to measure $\sim 0.1-0.3 \mathrm{pc}$ in size. Panel (B) shows the "ladder" region, which shows prominent ionized gas emission (blue) on the edges of the dusty clouds. Both panels were created using the same three-color map as Figure 5.

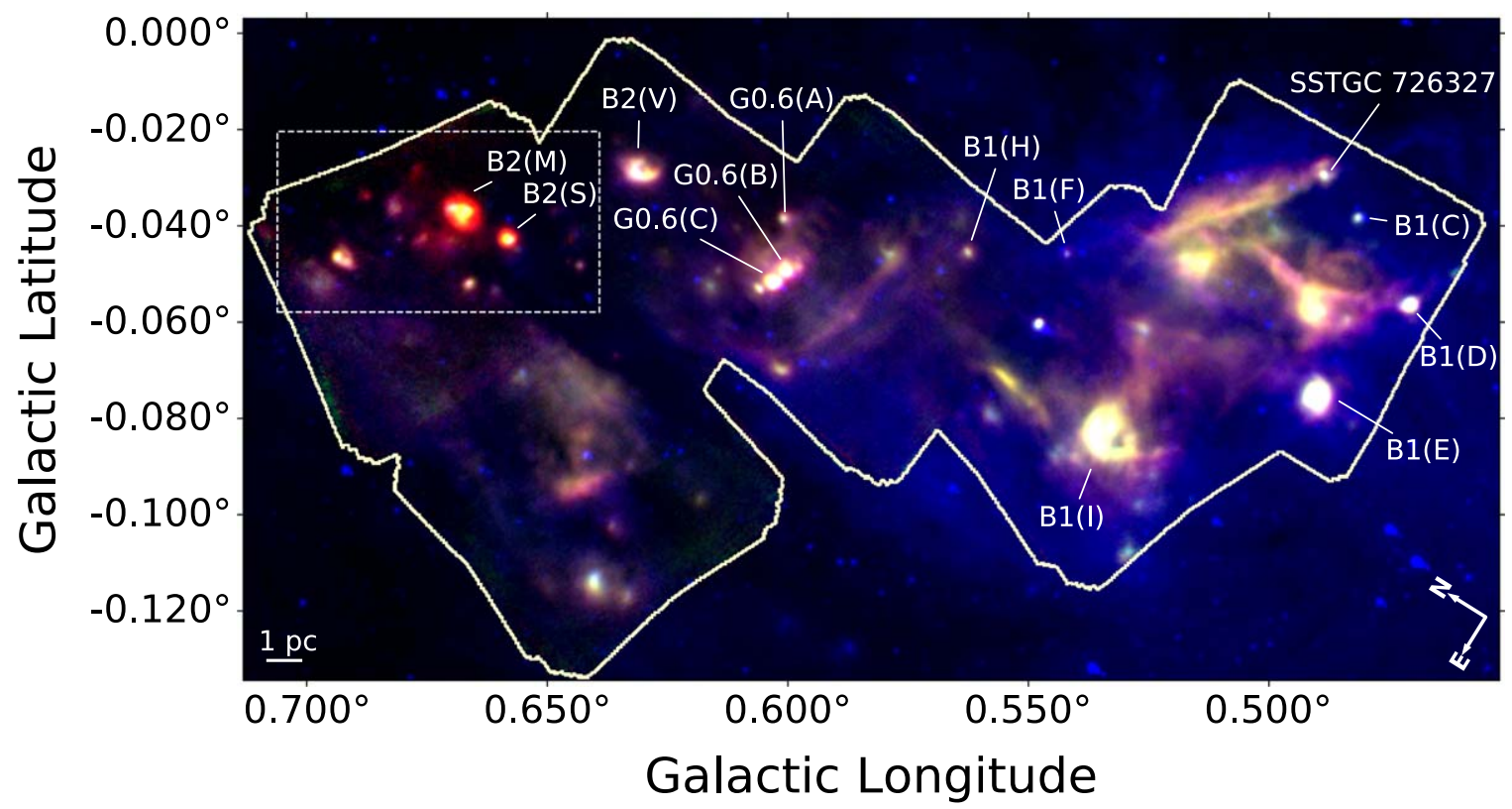

Figure 7. False-color map of the Sgr B complex using Spitzer/IRAC $8 \mu \mathrm{m}$ (blue), SOFIA/FORCAST $25 \mu \mathrm{m}$ (green), and $37 \mu \mathrm{m}$ (red) data. We use the IRAC $8 \mu \mathrm{m}$ data in this figure rather than the HST Paschen- $\alpha$ survey because the Sgr B region is outside of the HST survey footprint. Sources of interest are labeled throughout the region following the naming convention of Mehringer et al. $(1992,1993)$ with the exception of Sgr B2 Main, Sgr B2 South, and Sgr B1(A), which is associated with the massive YSO SSTGC 726327. Labels for sources in the G0.6-0.0 region are abbreviated "G0.6." The dashed box shows a region of the map that is featured in Figure 8, and the footprint of the SOFIA/FORCAST survey is also shown as a solid white outline for reference.

only a few of the massive stars belonging to the cluster (Figure 5, lower panel). Our survey observations also show dust associated with two extended nebulae surrounding the candidate luminous blue variable (LBV) stars, the Pistol star and G0.120-0.048 (Lau et al. 2014a). While there is a third known LBV candidate in this region, qF362 (Figer et al. 1999), there is no obvious infrared counterpart associated with this source in the FORCAST maps. The data obtained as part of our survey of the Sickle region will be explored in depth in a subsequent paper focused on the finger-like structures and other prominent features of the $\mathrm{H}$ II region discussed in this section.

\subsection{Sgr B1 and Sgr B2 Complexes}

The Sgr B complex is the easternmost region mapped in our survey. Sgr B is located along the galactic plane with a projected separation between $\sim 16^{\prime}$ and $23^{\prime}$ ( $\sim 40-50 \mathrm{pc}$ ) from Sgr A as measured from the near and far sides of Sgr B. This complex is most frequently discussed in terms of three distinct parts: Sgr B1, Sgr B2, and G0.6-0.0, which lies between Sgr B1 and Sgr B2 (Figure 7). Sgr B2 is one of the most massive and active star-forming regions in our Galaxy, having a few hundred sources that are likely a mix of young stellar objects and slightly more developed stars that have produced $\mathrm{H}$ II 


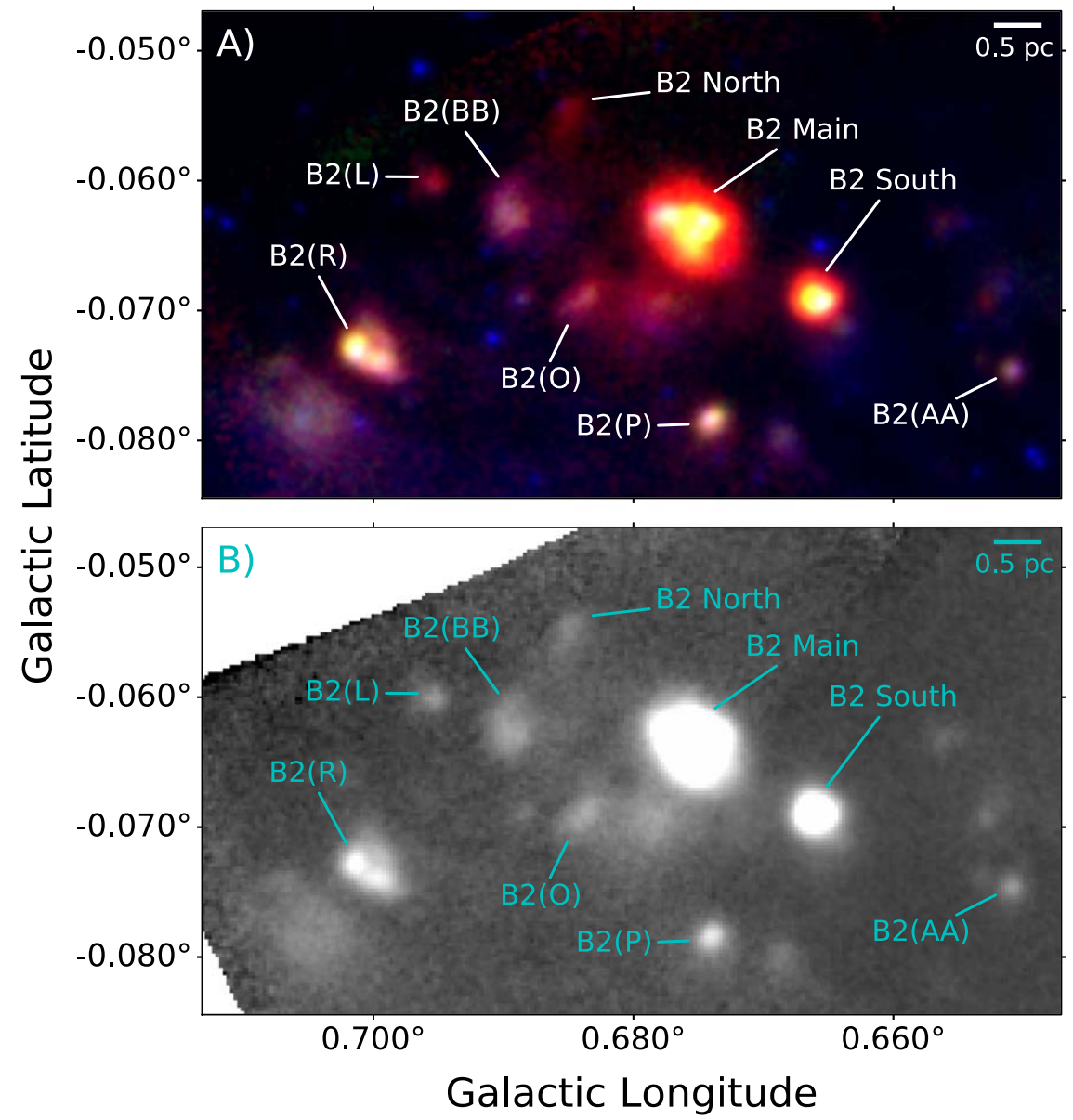

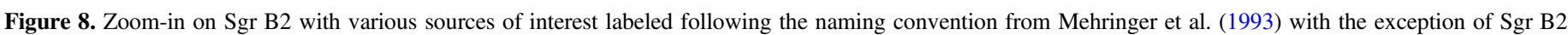

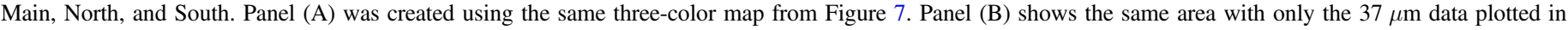
grayscale.

regions (de Pree et al. 1995, 1996; Ginsburg et al. 2018). In contrast, Sgr B1 is a more evolved star-forming region kinematically linked to Sgr B2, which contains a population of less extinct (embedded) H II regions (Mehringer et al. 1992, 1993; Lang et al. 2010). There is, however, recent evidence to suggest that these $\mathrm{H}$ II regions are a result of evolved massive stars passing through the dense medium in Sgr $\mathrm{B} 1$, as opposed to young $\mathrm{O} / \mathrm{B}$ stars that have formed within the cloud (Simpson et al. 2018).

The FORCAST observations of Sgr B reveal a number of interesting features throughout the region. Qualitatively, Sgr B1 shows considerable extended emission with large filamentary structures, shells, and bubbles, most of which have radio counterparts, while Sgr B2 appears as a collection of compact reddened sources. A few of the brightest mid-infrared sources in this region include the well-known complexes Sgr B2 Main and Sgr B2 South as well as Sgr B2 V (e.g., Etxaluze et al. 2013). Near Sgr B2 Main and South, we note several relatively faint IR sources that appear to be counterparts to known compact and ultracompact $\mathrm{H}$ II regions (Figure 8). The presence of these sources provides further evidence for recent star formation activity outside of the brightest complexes in Sgr B2, which is consistent with recent ALMA observations that show evidence of extended star formation outside of the main clusters in Sgr B2 (Ginsburg et al. 2018). Study of the brightest and faintest mid-IR sources in this region will require use of both the FORCAST data set and the Spitzer/MIPS $24 \mu \mathrm{m}$ data.
As part of a future data release, we are planning to create a combined map between these two data sets that will allow for study of regions like Sgr B2 where a large dynamic range is needed to effectively dissect the region.

Although the FORCAST data of Sgr B1 do show a bright, compact source coincident with one of the massive YSOs (SSTGC 726327) identified in An et al. (2011), the other candidates from that paper within our observations are unremarkable. There is, however, a strong ridge of warm dust emission immediately adjacent to one of the Wolf-Rayet stars found in Mauerhan et al. (2010) that does not have a strong radio counterpart and might be evidence of the effect of a orbiting massive star not formed in situ. Simpson et al. (2018) suggested, based on maps of the [O III] 52 and $88 \mu \mathrm{m}$ lines in Sgr B1 with FIFI-LS, that the region may not, in fact, be forming stars as we see in Sgr B2, but rather the observed emission may be the result of passing massive stars, such as is seen in the regions surrounding the Arches and Quintuplet clusters.

\subsection{Sgr C and Neighbors}

The Sgr C complex is the westernmost region mapped in our survey. Sgr $\mathrm{C}$ is located along the Galactic plane with a projected separation of $\sim 15^{\prime}(\sim 35 \mathrm{pc})$ from Sgr A. Our observations of Sgr $\mathrm{C}$ focused on the main Sgr C H II region with an additional adjacent pointing to the west containing the position of source " $\mathrm{C}$ " from Liszt \& Spiker (1995). Earlier measurements of ionized and 


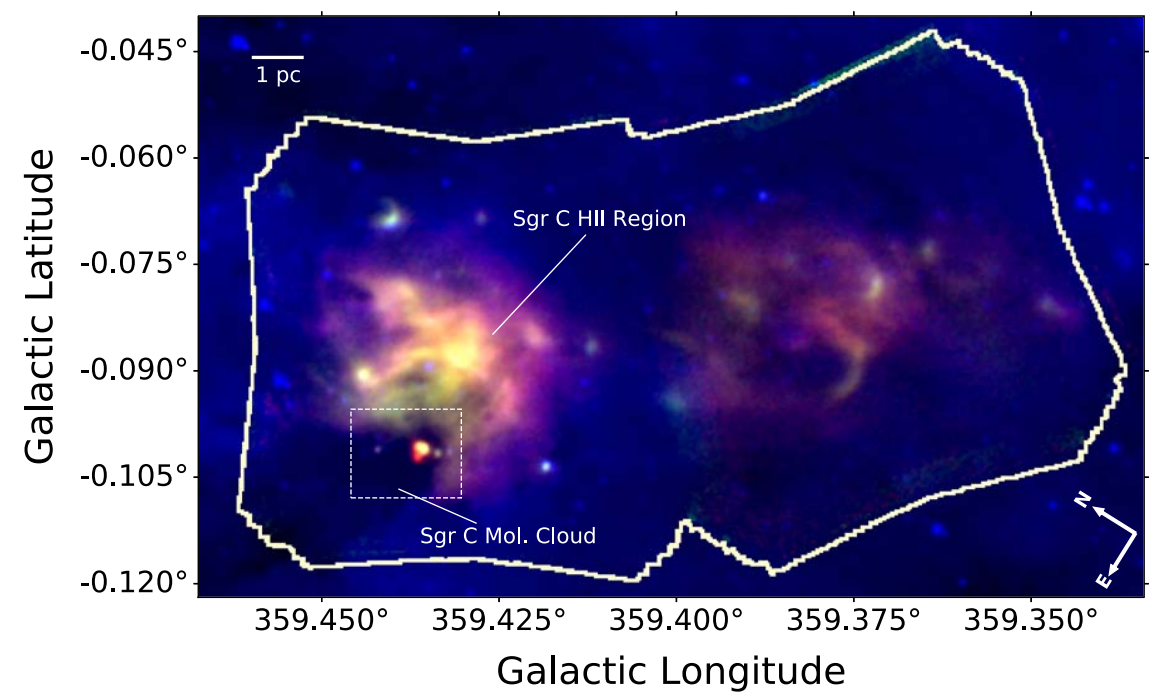

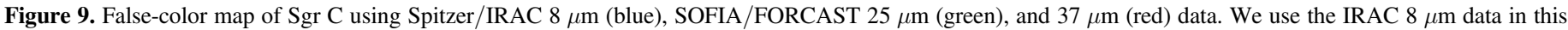

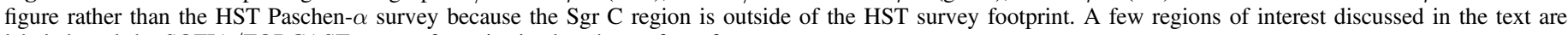
labeled, and the SOFIA/FORCAST survey footprint is also shown for reference.

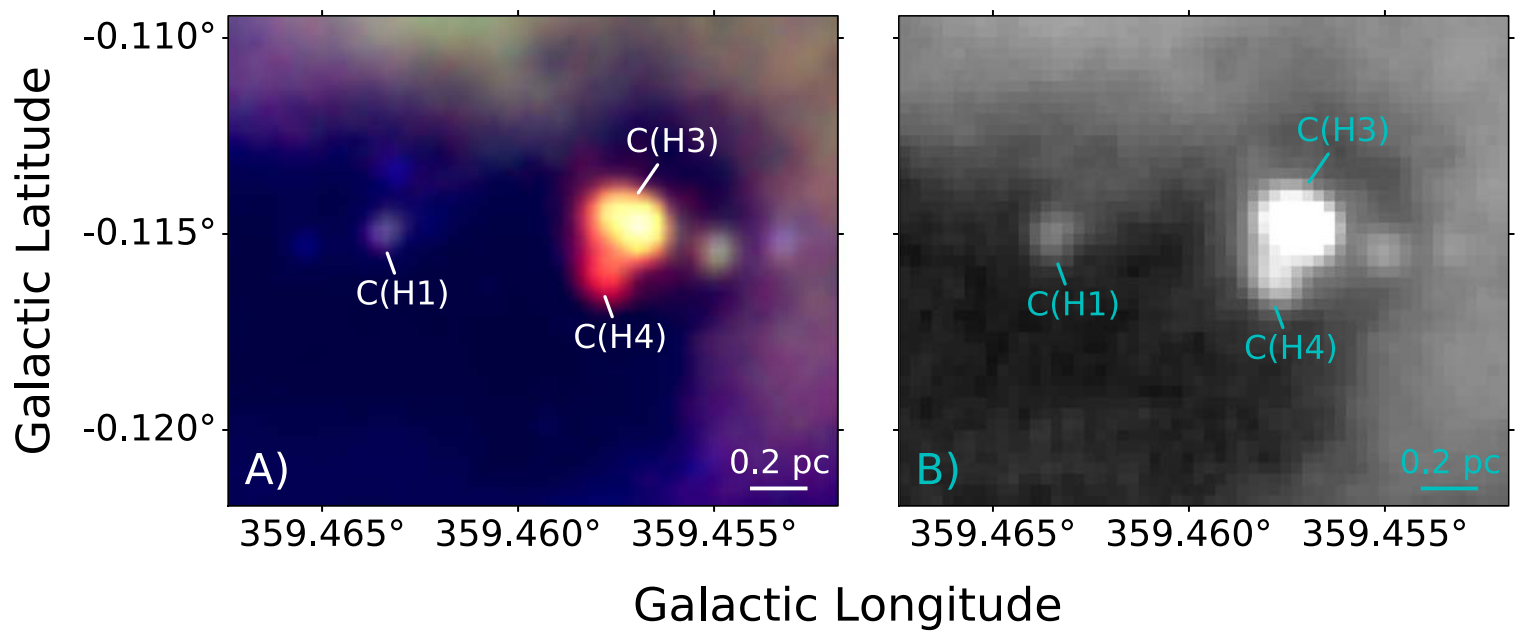

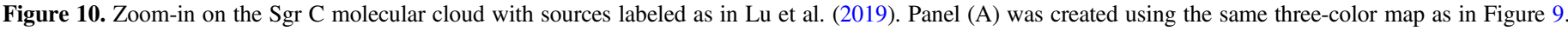
Panel (B) shows the same area using the $37 \mu \mathrm{m}$ data in grayscale.

molecular gas suggest that the $\mathrm{H}$ II region has a shell-like morphology that is likely created by massive stars that have blown out a cavity in the surrounding gas (Lang et al. 2010). The brightest portions of the extended 25 and $37 \mu \mathrm{m}$ emission we observe in this region are associated with the Sgr C H II region (Figure 9). However, the morphology of the dust appears quite complex compared to a simple shell configuration.

We also observe the Sgr C Molecular Cloud as an infrareddark cloud at 25 and $37 \mu \mathrm{m}$. The molecular cloud has a velocity of $-55 \mathrm{~km} \mathrm{~s}^{-1}$ (Kendrew et al. 2013), indicating that it is likely associated with the $\mathrm{H}$ II region, which has a measured velocity of $-65 \mathrm{~km} \mathrm{~s}^{-1}$ from recombination line emission (Lang et al. 2010). Toward the dark cloud, we find a number of bright midinfrared sources (Figure 10). The most luminous of these sources ( $\mathrm{Sgr} \mathrm{C} \mathrm{H3)} \mathrm{is} \mathrm{reminiscent} \mathrm{of} \mathrm{the} \mathrm{bright,} \mathrm{red} \mathrm{sources} \mathrm{we}$ observe in Sgr B2. This object has been previously cataloged as an ultracompact H II region by Forster \& Caswell (2000), and Kendrew et al. (2013) show that this source has an additional substructure, including two dusty protostellar cores and signatures of an outflow that are indicative of ongoing highmass star formation. There are two additional infrared sources near Sgr $\mathrm{C} \mathrm{H} 3$, which are also cataloged as $\mathrm{H}$ II regions in $\mathrm{Lu}$ et al. (2019) and can be found in Figure 10.

The Sgr C complex is also known for its prominent nonthermal filament (NTF; e.g., Roy 2003). Our survey did not cover the location of the filament because of our focus on the mid-infrared bright regions. However, the NTF should be discussed along with the $\mathrm{H}$ II region, because of the likely association of these features. In fact, large $\mathrm{H}$ II regions may be key to producing bright nonthermal features at radio wavelengths by providing a vast supply of free electrons that are accelerated to relativistic velocities via locally strong magnetic fields (Serabyn \& Morris 1994; Uchida et al. 1996). There are numerous similarities between the well-known GC Radio Arc and the Sgr C NTF that warrant further study. This is another area where combining the MIPS and FORCAST data is needed to create a more complete picture of this region in the infrared and improve our understanding of the environments that give rise to prominent radio features. Certainly this will be an interesting area to study with the release of high-level data products from this survey. 


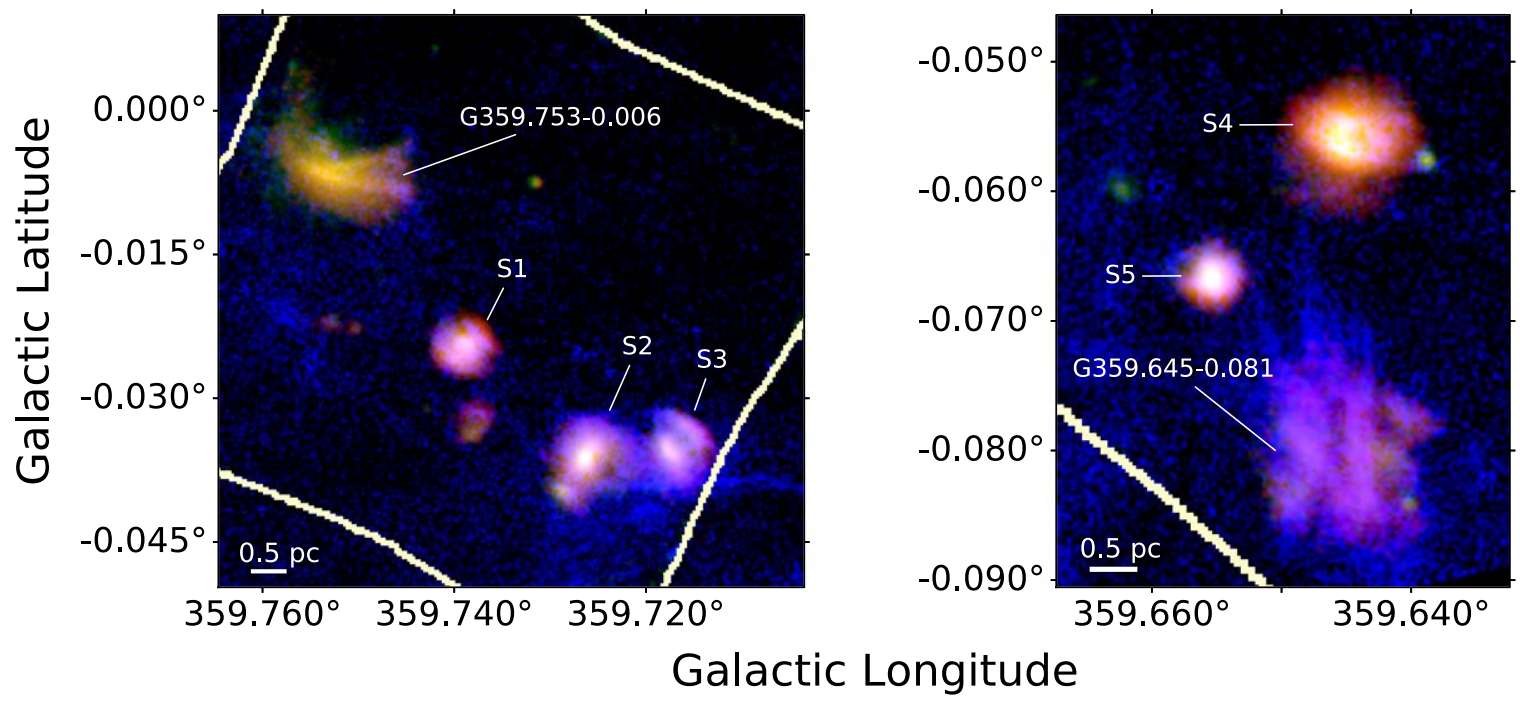

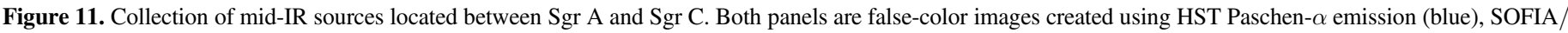

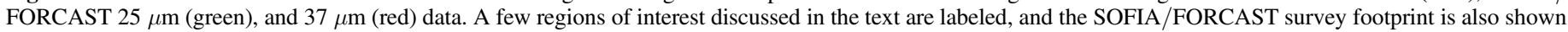
for reference. These less well studied sources are discussed in Section 3.5.

Between Sgr A and Sgr C, there is a smattering of compact mid-infrared sources that have not garnered as much attention as other portions of the GC. These sources appear to be in somewhat isolated environments, which speaks to the apparent dichotomy between regions located at negative and positive Galactic longitudes, the latter of which are thought to be more actively forming stars (Longmore et al. 2013; Barnes et al. 2017). These isolated western sources are of interest because several are cataloged as candidate YSOs from the ISOGAL survey (Immer et al. 2012). Our observations of a handful of these sources show a diverse range of morphologies and colors. For example, G359.753-0.006 shows a prominent bow-shock-like morphology (Figure 11). Several other nearby sources (G359.738-0.024, G359.726-0.037, G359.716-0.035, G359.64477-0.056, and G359.655 -0.067 , labeled respectively as S1, S2, S3, S4, and S5 in Figure 11) are more compact but still resolved in the FORCAST observations. These sources each show ionized gas emission and are possibly compact or ultracompact H II regions. Interestingly, S2 and S3 may be interacting with a nearby ionized gas filament that is visible in the Paschen- $\alpha$ data, and this collection of sources may have similarities to the G359.866+0.002 complex discussed in Section 3.2.

One of the most interesting sources in this region is G359.645-0.081, which is a relatively faint extended source. It is brighter at $37 \mu \mathrm{m}$ compared to $25 \mu \mathrm{m}$, indicating that it is somewhat cool and is plausibly the edge of a molecular cloud. However, the amount of extended ionized gas emission in addition to the relatively cool dust emission is somewhat peculiar compared to other extended sources in our survey. Prior observations of this region with Spitzer/IRS show a few high-ionization species including [Ne V] $24.32 \mu \mathrm{m}$ and [O IV] $25.9 \mu \mathrm{m}$ near this position (Simpson 2018), indicative of a hard ionization source. The morphology and excitation of G359.645 -0.081 is somewhat reminiscent of the Sgr A East ejecta (Lau et al. 2015) and also the supernova remnant G292.0+1.8 (Ghavamian et al. 2009); however, this region lacks an X-ray component (Simpson 2018), suggesting it is not a supernova remnant. Considering both the unusual color and ionization properties, this source merits further study.

\section{Summary}

In this paper, we have presented observations and initial results from the SOFIA/FORCAST survey of the GC. Our survey focused on some of the brightest infrared regions in the GC that trace recent star formation. These data provide the highest spatial resolution mapping of the Galactic Center at 25 and $37 \mu \mathrm{m}$ to date (FWHM $\sim 2$ !" 3 and FWHM $\sim 3$ !" 4, respectively), and they cover several interesting regions that were badly saturated in the Spitzer/MIPS $24 \mu \mathrm{m}$ data. Ultimately, our primary science objective for the survey is to better characterize star formation in the GC, in particular the well-known SFR discrepancy in this region. Examining this topic will require high-level data products, including source catalogs and enhanced maps that will be produced as a part of this program and released to the broader astronomical community in a future data release.

We have produced this paper to describe the survey plan, observations, and initial highlights from the data as an accompaniment to the initial survey data release. All data collected from this survey, including Level 3 and 4 data products, can be found on the SOFIA DCS and IRSA. In this work, we have presented short summaries of several featured regions and sources, including extended structures near the CND, the Arched Filaments and Sickle H II regions, and embedded star formation in Sgr B2 and Sgr C. While primarily qualitative, these case studies illustrate the scope and utility of the survey data. We are planning future studies around several of these topics, which will be presented in later works or possibly accompany future data releases.

We thank the anonymous referee for their comments that improved the quality of this paper. Additionally, we thank the USRA Science and Mission Ops teams and the entire SOFIA staff for making this survey possible. In particular, we thank Mike Gordon and Jim De Buizer, who helped to support our observations. Additionally, we thank the many people who have worked on FORCAST over the years, including but not limited to George Gull, Justin Schoenwald, Chuck Henderson, Joe Adams, and Andrew Helton. Financial support for this work was provided by NASA through award number 
NNA17BF53C issued by USRA. This material is based upon work supported by the National Science Foundation under grant No. AST-1813765. A.T.B. would like to acknowledge the funding provided from the European Union's Horizon 2020 research and innovation programme (grant agreement No. 726384).

This work is based on observations made with the NASA/DLR Stratospheric Observatory for Infrared Astronomy (SOFIA). SOFIA science mission operations are conducted jointly by the Universities Space Research Association, Inc. (USRA) under NASA contract NAS2-97001, and the Deutsches SOFIA Institut (DSI) under DLR contract 50 OK 0901. Financial support for FORCAST was provided by NASA through award 8500-98-014 issued by USRA.

This work made use of data products from the Spitzer Space Telescope, which is operated by the Jet Propulsion Laboratory, California Institute of Technology, under a contract with NASA. Additionally, this research made use of data products from the Midcourse Space Experiment. Processing of the data was funded by the Ballistic Missile Defense Organization with additional support from NASA Office of Space Science. This research has also made use of the NASA/IPAC Infrared Science Archive, which is operated by the Jet Propulsion Laboratory, California Institute of Technology, under contract with the National Aeronautics and Space Administration. Finally, this research has also made use of the VizieR catalog access tool, CDS, Strasbourg, France. The original description of the VizieR service was published in Ochsenbein et al. (2000).

\section{ORCID iDs}

Matthew J. Hankins (1 https://orcid.org/0000-0001-9315-8437 Mark R. Morris (10 https://orcid.org/0000-0002-6753-2066 Elisabeth A. C. Mills (ib https://orcid.org/0000-0001-8782-1992 Janet P. Simpson (1) https://orcid.org/0000-0001-8095-4610 Terry L. Herter (i) https://orcid.org/0000-0002-3856-8385 Steven N. Longmore (1) https://orcid.org/0000-0001-6353-0170 John Bally (1) https://orcid.org/0000-0001-8135-6612 Mansi M. Kasliwal (1) https://orcid.org/0000-0002-5619-4938

\section{References}

An, D., Ramírez, S. V., Sellgren, K., et al. 2011, ApJ, 736, 133 Bally, J., Stark, A. A., Wilson, R. W., \& Henkel, C. 1987, ApJS, 65, 13 Barnes, A. T., Longmore, S. N., Avison, A., et al. 2019, MNRAS, 486, 283 Barnes, A. T., Longmore, S. N., Battersby, C., et al. 2017, MNRAS, 469, 2263 Calzetti, D., Kennicutt, R. C., Engelbracht, C. W., et al. 2007, ApJ, 666, 870 Churchwell, E., Babler, B. L., Meade, M. R., et al. 2009, PASP, 121, 213 Clarke, M., Vacca, W. D., \& Shuping, R. Y. 2015, in ASP Conf. Ser. 495, Redux: A Common Interface for SOFIA Data Reduction Pipelines, ed. A. R. Taylor \& E. Rosolowsky (San Francisco, CA: ASP), 355

Cotera, A., Stolovy, S., Ramirez, S., et al. 2006, JPhCS, 54, 183

Cotera, A. S., Erickson, E. F., Colgan, S. W. J., et al. 1996, ApJ, 461, 750

de Pree, C. G., Gaume, R. A., Goss, W. M., \& Claussen, M. J. 1995, ApJ, 451,284

de Pree, C. G., Gaume, R. A., Goss, W. M., \& Claussen, M. J. 1996, ApJ, 464, 788

Dong, H., Lacy, J. H., Schödel, R., et al. 2017, MNRAS, 470, 561

Dong, H., Wang, Q. D., \& Morris, M. R. 2012, MNRAS, 425, 884

Egan, M., Price, S., Kraemer, K., et al. 2003, Air Force Research Laboratory

Technical Report AFRL-VS-TR-2003-1589, https://irsa.ipac.caltech.edu/ Missions/msx.html

Etxaluze, M., Goicoechea, J. R., Cernicharo, J., et al. 2013, A\&A, 556, A137 Figer, D. F. 2009, in Massive Stars: From Pop III and GRBs to the Milky Way, ed. M. Livio \& E. Villaver (Cambridge: Cambridge Univ. Press), 40

Figer, D. F., Kim, S. S., Morris, M., et al. 1999, ApJ, 525, 750

Forster, J. R., \& Caswell, J. L. 2000, ApJ, 530, 371

Fritz, T. K., Gillessen, S., Dodds-Eden, K., et al. 2011, ApJ, 737, 73
Ghavamian, P., Raymond, J. C., Blair, W. P., et al. 2009, ApJ, 696, 1307 Ginsburg, A., Bally, J., Barnes, A., et al. 2018, ApJ, 853, 171

Guesten, R., \& Henkel, C. 1983, A\&A, 125, 136

Guesten, R., Walmsley, C. M., Ungerechts, H., \& Churchwell, E. 1985, A\&A, 142,381

Habibi, M., Stolte, A., \& Harfst, S. 2014, A\&A, 566, A6

Hankins, M. J., Lau, R. M., Mills, E. A. C., Morris, M. R., \& Herter, T. L. 2019, ApJ, 877, 22

Hankins, M. J., Lau, R. M., Morris, M. R., \& Herter, T. L. 2017, ApJ, 837, 79 Hankins, M. J., Lau, R. M., Morris, M. R., et al. 2016, ApJ, 827, 136 Herter, T. L., Adams, J. D., De Buizer, J. M., et al. 2012, ApJL, 749, L18

Herter, T. L., Vacca, W. D., Adams, J. D., et al. 2013, PASP, 125, 1393 Hinz, J., Rieke, G., Yusef-Zadeh, F., et al. 2009, ApJS, 181, 227

Immer, K., Schuller, F., Omont, A., \& Menten, K. M. 2012, A\&A, 537, A121 Kendrew, S., Ginsburg, A., Johnston, K., et al. 2013, ApJL, 775, L50

Krabbe, A., Genzel, R., Drapatz, S., \& Rotaciuc, V. 1991, ApJL, 382, L19

Krabbe, A., Genzel, R., Eckart, A., et al. 1995, ApJL, 447, L95

Kruijssen, J. M. D., \& Longmore, S. N. 2013, MNRAS, 435, 2598

Lada, C. J., Forbrich, J., Lombardi, M., \& Alves, J. F. 2012, ApJ, 745, 190

Lang, C. C., Goss, W. M., Cyganowski, C., \& Clubb, K. I. 2010, ApJS, 191,275

Lang, C. C., Goss, W. M., \& Morris, M. 2001, AJ, 121, 2681

Lang, C. C., Goss, W. M., \& Morris, M. 2002, AJ, 124, 2677

Lang, C. C., Goss, W. M., \& Wood, O. S. 1997, ApJ, 474, 275

Lau, R. M., Hankins, M. J., Herter, T. L., et al. 2016, ApJ, 818, 117

Lau, R. M., Herter, T. L., Morris, M. R., \& Adams, J. D. 2014a, ApJ, 785, 120

Lau, R. M., Herter, T. L., Morris, M. R., \& Adams, J. D. 2014b, ApJ, 794, 108

Lau, R. M., Herter, T. L., Morris, M. R., Becklin, E. E., \& Adams, J. D. 2013, ApJ, 775, 37

Lau, R. M., Herter, T. L., Morris, M. R., Li, Z., \& Adams, J. D. 2015, Sci, 348,413

Lee, E. J., Murray, N., \& Rahman, M. 2012, ApJ, 752, 146

Liszt, H. S., \& Spiker, R. W. 1995, ApJS, 98, 259

Liu, H. B., Hsieh, P.-Y., Ho, P. T. P., et al. 2012, ApJ, 756, 195

Longmore, S. N., Bally, J., Testi, L., et al. 2013, MNRAS, 429, 987

Lu, J. R. 2018, in The Birth of Star Clusters, Astrophysics and Space Science Library, Vol. 424, ed. S. Stahler (Cham: Springer), 69

Lu, X., Zhang, Q., Kauffmann, J., et al. 2019, ApJ, 872, 171

Mauerhan, J. C., Cotera, A., Dong, H., et al. 2010, ApJ, 725, 188

Mehringer, D. M., Palmer, P., Goss, W. M., \& Yusef-Zadeh, F. 1993, ApJ, 412, 684

Mehringer, D. M., Yusef-Zadeh, F., Palmer, P., \& Goss, W. M. 1992, ApJ, 401, 168

Mills, E. A. C. 2017, arXiv:1705.05332

Molinari, S., Swinyard, B., Bally, J., et al. 2010, PASP, 122, 314

Morris, M., Polish, N., Zuckerman, B., \& Kaifu, N. 1983, AJ, 88, 1228

Morris, M., \& Serabyn, E. 1996, ARA\&A, 34, 645

Muno, M. P., Bower, G. C., Burgasser, A. J., et al. 2006, ApJ, 638, 183

Nagata, T., Woodward, C. E., Shure, M., Pipher, J. L., \& Okuda, H. 1990, ApJ, 351,83

Najarro, F., Geballe, T. R., Figer, D. F., \& de la Fuente, D. 2017, ApJ, 845, 127

Ochsenbein, F., Bauer, P., \& Marcout, J. 2000, A\&AS, 143, 23

Okuda, H., Shibai, H., Nakagawa, T., et al. 1990, ApJ, 351, 89

Ponti, G., Morris, M. R., Terrier, R., et al. 2015, MNRAS, 453, 172

Ramírez, S. V., Arendt, R. G., Sellgren, K., et al. 2008, ApJS, 175, 147

Reid, M. J. 1993, ARA\&A, 31, 345

Robitaille, T. P., Whitney, B. A., Indebetouw, R., Wood, K., \& Denzmore, P. 2006, ApJS, 167, 256

Roy, S. 2003, A\&A, 403, 917

Scoville, N. Z., Solomon, P. M., \& Penzias, A. A. 1975, ApJ, 201, 352

Serabyn, E., \& Morris, M. 1994, ApJL, 424, L91

Shahzamanian, B., Schödel, R., Nogueras-Lara, F., et al. 2019, A\&A, 632, A116

Simpson, J. P. 2018, ApJ, 857, 59

Simpson, J. P., Colgan, S. W. J., Cotera, A. S., Kaufman, M. J., \& Stolovy, S. R. 2018, ApJL, 867, L13

Steinke, M., Oskinova, L. M., Hamann, W.-R., et al. 2016, A\&A, 588, A9

Stolte, A., Grebel, E. K., Brandner, W., \& Figer, D. F. 2002, A\&A, 394, 459

Tuthill, P., Monnier, J., Tanner, A., et al. 2006, Sci, 313, 935

Uchida, K. I., Morris, M., Serabyn, E., \& Guesten, R. 1996, ApJ, 462, 768

Wang, Q. D., Dong, H., Cotera, A., et al. 2010, MNRAS, 402, 895

Young, E. T., Becklin, E. E., Marcum, P. M., et al. 2012, ApJL, 749, L17

Yusef-Zadeh, F., Hewitt, J. W., Arendt, R. G., et al. 2009, ApJ, 702, 178

Yusef-Zadeh, F., Lacy, J. H., Wardle, M., et al. 2010, ApJ, 725, 1429

Yusef-Zadeh, F., \& Morris, M. 1987, ApJ, 320, 545

Zhao, J.-H., Morris, M. R., \& Goss, W. M. 2016, ApJ, 817, 171 